\title{
A BGK model for reactive mixtures of polyatomic gases with continuous internal energy
}

\author{
M. Bisi ${ }^{a}$, R. Monaco $^{b)}$ A.J. Soares ${ }^{c}$ \\ ${ }^{a)}$ Dipartimento di Scienze Matematiche, Fisiche e Informatiche, Università di Parma, Parma, Italy, marzia.bisi@unipr.it \\ b) DIST, Politecnico di Torino, Torino, Italy, roberto.monaco@polito.it \\ ${ }^{c)}$ Centro de Matemática, Universidade do Minho, Braga, Portugal, ajsoares@math.uminho.pt
}

(January 2018)

\begin{abstract}
In this paper we derive a BGK relaxation model for a mixture of polyatomic gases with a continuous structure of internal energies. The emphasis of the paper is on the case of a quaternary mixture undergoing a reversible chemically reaction of bimolecular type. For such a mixture we prove an $\mathcal{H}$ theorem and characterize the equilibrium solutions with related mass action law of chemical kinetics. Further, a Chapman-Enskog asymptotic analysis is performed in view of computing the first-order non-equilibrium corrections to the distribution functions and investigating the transport properties of the reactive mixture. The chemical reaction rate is explicitly derived at the first order and the balance equations for the constituent number densities are derived at the Euler level.
\end{abstract}

Keywords: Polyatomic gas mixtures, Chemical reactions, Boltzmann equation, BGK models.

MSC 2010: 82C40, 76P05, 80A32

PACS: $47.70 . \mathrm{Fw}, 47.45 . \mathrm{Ab}, 31.15 . \mathrm{vq}$

\section{Introduction}

The investigation of multicomponent flows undergoing chemical reactions is motivated by several engineering applications, as it is well documented in literature, see for example [13, 18]. In particular, reactive mixtures of polyatomic gases appear in many problems for which the impact of the internal energies is relevant [21]. In many applications, an hydrodynamic framework is enough to describe the essential part of the problem and the derivation of the fluid dynamical equations from the kinetic theory of gases can give a major contribution. In fact, the reactive production terms and the transport coefficients appearing in the equations can be explicitly evaluated from the detailed dynamics considered in the kinetic model. Several studies in this direction have been advanced in the recent years, based on the Boltzmann equation as well as on its BGK approximation. Among others, we quote here the papers [12, 15, 20] in the context of the Boltzmann equation, and $[2,9,16,17]$ with reference to the BGK model.

In kinetic theory, polyatomic gases are modelled by means of a suitable internal energy, in order to mimic non-translational degrees of freedom. Such additional internal energy may be assumed a discrete or a continuous variable and plays an important role in the the model dynamics. In aligment with this framework, a Boltzmann model for a quaternary reactive mixture whose constituents have a fixed internal energy (thus they are monatomic gases) has been proposed in [20] and then extended in [15] to a mixture whose constituents have various (discrete) degrees of internal energies. Paper [12] considers a reactive mixture of polyatomic gases described in terms of a continuous energy variable and derive a Boltzmann model for such a mixture following the so-called Borgnakke-Larsen procedure [8]. Even paper [14], dealing with a dissociation-recombination problem, assumes continuous internal energy for the excited diatomic molecules. 
Concerning the BGK approaches for chemically reactive mixtures, papers [9] and [16] construct a BGK approximating model for the Boltzmann system of paper [20]. The main difference between them is that the model of paper [9] splits the relaxation operator of each species in a mechanical operator and a reactive operator, whereas the model of paper [16] approximates together both reactive and non-reactive Boltzmann operators by a single relaxation term. Another strategy is followed in paper [17], where a relaxation BGK model is proposed, approximating separately all individual elastic collision terms and the reactive collision term, so that the resulting BGK model retains the influence of the chemical reaction but also the mixture effects. Finally, paper [2] builds up a consistent model for both an inert and a reactive mixture of polyatomic gases with a set of discrete internal energy levels, and then in $[5,7]$ suitable hydrodynamic limits are investigated in inert frames, comparing the results with the ones obtained by the phenomenological Extended Thermodynamics.

In the present work, we follow the line of paper [2] but we assume a continuous internal energy variable. We consider a mixture of polyatomic gases and build up a consistent BGK approximation of the Boltzmann model proposed in paper [12], both for an inert mixture and for a chemically reactive mixture. The presentation of the inert case serves as a basis for the more complicated reactive frame, in the sense that it becomes easier to identify additional issues appearing from the chemical reaction. The model is constructed replacing the complete Boltzmann collision operator of each species by only one relaxation term. Such term is based on a Gaussian attractor whose macroscopic parameters are univocally characterized by imposing that collision invariants are the same in the Boltzmann system and in the proposed BGK model. As a consequence, the BGK model is consistent with the correct conservation laws. In comparison to the exact Boltzmann system, but also to the BGK model of paper [17], our model in this paper presents a rather simple collision term. In view of exploiting such simplicity, we study the hydrodynamic limit of a reactive mixture with the main objective of determining the first-order non-equilibrium corrections to the distribution functions and investigating the transport properties. We restrict the analysis in this paper to the reactive production terms and to the number densities of the constituents. Our BGK model, which describes in a unique collision operator elastic and reactive effects, allows us to investigate a fast reactive regime, while the asymptotic limit considered in [12] deals with a slow chemical reaction, with the dominant role in the kinetic equations played by the elastic Boltzmann operators.

After this introduction, the paper proceeds as follows. In Section 2 we summarize the main aspects of the kinetic model of Boltzmann type proposed in paper [12] for a mixture of polyatomic gases with a continuous internal energy variable. In Section 3, starting from the model of paper [12], we derive our BGK relaxation model for an inert mixture of polyatomic gases with an arbitrary number of constituents and a continuous structure of internal energies. The macroscopic parameters characterizing the Gaussian attractors are univocally determined. Then, in Section 4, we consider a quaternary reactive mixture of polyatomic gases undergoing a reversible chemical reaction of bimolecular type. The previous BGK model is extended to such a mixture introducing one approximating operator for each species as well, and thus incorporating together both non-reactive and reactive collision operators of the original Boltzmann model. The Gaussian attractors are univocally characterized and the mass action law of the chemical kinetics plays now an important role in the construction of the BGK approximation. The trend to equilibrium of such a model is investigated, and an $\mathcal{H}$-theorem is proven in spatially homogeneous conditions. Finally, in Section 5 we deal with the hydrodynamic limit of the BGK model of Section 4. Starting from a convenient scaling of the kinetic equations compatible with a chemical regime of fast reaction, we use the Chapman-Enskog method to determine the first-order corrections to the distribution functions. The transport properties of the reactive mixture are investigated with reference in particular to number densities of single constituents, which are not preserved by chemical reaction. Balance equations for them are derived at Euler level, including also the chemical production rate, which involves the firstorder perturbations of the densities themselves. The paper ends with our conclusions and some final remarks in Section 6. 


\section{Preliminaries}

By following the line proposed in [12], we consider a mixture of $N$ polyatomic gases (species) and to each one we associate an index $i=1, \ldots, N$. For each species, the particle mass is denoted by $m^{i}$, and the distribution function $f^{i}$ is assumed to depend also on an internal energy variable $I$, to describe nontranslational degrees of freedom. Species macroscopic variables are reconstructed as suitable moments of the distribution function $f^{i}(\mathbf{v}, I)$; for instance, number density of each species is provided by

$$
n^{i}=\int_{\mathbb{R}^{3}} \int_{0}^{+\infty} f^{i}(\mathbf{v}, I) \varphi^{i}(I) d I d \mathbf{v},
$$

where $\varphi^{i}(I) d I$ is a non-negative measure which is a parameter of the model. In the case of polytropic gases, one usually takes the energy measure in the form

$$
\varphi^{i}(I)=I^{\alpha_{i}}, \quad \text { with } \quad \alpha_{i} \geq 0, \quad \text { for } \quad i=1, \ldots, n,
$$

where $\alpha_{i}$ is related to the number of degrees of freedom of particles [19]. The particular case $\alpha_{i}=0$ corresponds to diatomic molecules. Moreover the total numerical and mass densities of the mixture are given by

$$
n=\sum_{i=1}^{N} n^{i}, \quad \rho=\sum_{i=1}^{N} m^{i} n^{i} .
$$

The distribution function $f^{i}(\mathbf{v}, I)$ admits two hierarchies of moments [19], one with respect to the molecular velocity $\mathbf{v}$ and another one with respect to the energy variable $I$. Analogous integrals to that of Eq. (1) provide classical species moments as mean velocity $\mathbf{u}^{i}$ and temperature $T^{i}$

$$
\mathbf{u}^{i}=\frac{1}{n^{i}} \int_{\mathbb{R}^{3}} \int_{0}^{+\infty} \mathbf{v} f^{i}(\mathbf{v}, I) \varphi^{i}(I) d I d \mathbf{v}, \quad T^{i}=\frac{m^{i}}{3 n^{i}} \int_{\mathbb{R}^{3}} \int_{0}^{+\infty}\left|\mathbf{v}-\mathbf{u}^{i}\right|^{2} f^{i}(\mathbf{v}, I) \varphi^{i}(I) d I d \mathbf{v},
$$

whereas suitable summation over the index $i$ provide global mixture moments, such as mean velocity $\mathbf{u}$ and temperature $T$,

$$
\mathbf{u}=\frac{1}{\rho} \sum_{i=1}^{N} m^{i} n^{i} \mathbf{u}^{i}, \quad T=\frac{1}{3 n} \sum_{i=1}^{N} m^{i} \int_{\mathbb{R}^{3}} \int_{0}^{+\infty}|\mathbf{v}-\mathbf{u}|^{2} f^{i}(\mathbf{v}, I) \varphi^{i}(I) d I d \mathbf{v} .
$$

As concerns the moments with respect to $I$, use will be made in the sequel especially of the first order moment

$$
\chi^{i}=\int_{\mathbb{R}^{3}} \int_{0}^{+\infty} f^{i}(\mathbf{v}, I) I \varphi^{i}(I) d I d \mathbf{v} .
$$

Let us recall that, starting from this framework, in the paper [12], following the so-called BorgnakkeLarsen procedure [8], kinetic equations have been derived both for an inert and for a reactive mixture of four polytropic gases undergoing a reversible bimolecular reaction. In each binary interaction, a fraction of the ingoing total energy is attributed to the internal energy of the outgoing pair of molecules and then randomly distributed between the two single particles. In spite of the heavy technicalities involved in the construction of the Boltzmann collision operators (skipped here, all details may be found in [12]), the proposed kinetic model is able to reproduce the expected conservation laws and long time evolution towards an equilibrium configuration. Both for inert and reactive mixtures, collision equilibria are provided by Maxwellian distributions with all species sharing the same mean velocity and temperature

$$
f_{M}^{i}(\mathbf{v}, I)=\frac{n^{i}}{q^{i}(T)}\left(\frac{m^{i}}{2 \pi T}\right)^{3 / 2} \mathrm{e}^{-\frac{1}{T}\left[\frac{m^{i}}{2}|\mathbf{v}-\mathbf{u}|^{2}+I\right]},
$$

where the denominator is given by

$$
q^{i}(T)=\int_{0}^{+\infty} \varphi^{i}(I) \mathrm{e}^{-I / T} d I
$$


and is the so-called "partition function", appearing also in kinetic models for polyatomic gases with discrete internal energies [5]. The total energy at the equilibrium state is provided by

$$
n \mathcal{E}=\sum_{i=1}^{N} \int_{\mathbb{R}^{3}} \int_{0}^{+\infty}\left(\frac{1}{2} m^{i}|\mathbf{v}|^{2}+I\right) f_{M}^{i}(\mathbf{v}, I) \varphi^{i}(I) d I d \mathbf{v}=\frac{1}{2} \rho|\mathbf{u}|^{2}+\frac{3}{2} n T+\sum_{i=1}^{N} n^{i} \frac{\eta^{i}(T)}{q^{i}(T)},
$$

where the last contribution is related to the internal energy levels of the molecules, with

$$
\eta^{i}(T)=\int_{0}^{+\infty} I \varphi^{i}(I) \mathrm{e}^{-I / T} d I
$$

For polytropic gases, with $\varphi^{i}(I)$ given by $(2)$, a simple integration by parts yields

$$
\eta^{i}(T)=\left(\alpha_{i}+1\right) T q^{i}(T),
$$

therefore the specific heat at constant volume, defined as $c_{v}(T)=\frac{\partial \mathcal{E}}{\partial T}$, turns out to be independent from $T$. Notice that the choice $\varphi^{i}(I)=1$, for $i=1, \ldots, n$, gives $c_{v}=\frac{5}{2}$, i.e. the correct caloric law for diatomic molecules. The classical value for monatomic gases, $c_{v}=\frac{3}{2}$, would correspond to the option $\alpha_{i}=-1$, for $i=1, \ldots, n$, but the partition function $q^{i}(T)$ is not finite for $\varphi^{i}(I)=I^{-1}$, therefore the kinetic model proposed in [12] is not suited for classical monatomic particles [19]. For reactive mixtures, equilibrium number densities and temperature have to fulfill a law of mass action, that will be explicitly recalled in Section 4. In [12] the authors prove that collision invariants for such a kinetic model are species number densities (or only proper combinations of them, in case of reactive mixtures), global momentum, and total energy, namely kinetic plus internal plus chemical (if available). Then, the hydrodynamic limit is performed under the hypothesis that the number of non-reactive collisions is much greater than the one of reactive collisions, providing nothing else than the Euler system of perfect and reactive gases. Moreover, in this context, an $\mathcal{H}$-theorem is proven and consequently the entropy inequality is obtained. For the BGK approximation that we will build up in the following sections, we will be able to consider asymptotic regimes with fast chemical reactions, and to push the hydrodynamic description to the next order of accuracy (at Navier-Stokes level), at least for what concerns distribution functions and chemical production rates appearing in balance equations for number densities.

\section{Inert mixture}

In this section we assume that the $N$ species are elastically scattering. In this frame, the model [12] has $N+4$ scalar collision invariants, corresponding to preservation of species number densities, global momentum, and total (kinetic plus internal) energy. Collision equilibria are provided by Maxwellian distributions (7) and (8).

We aim at building up a BGK model of the type originally proposed in [1] for inert mixtures, extended to reactive frames in [3] and then to polyatomic gases with discrete internal energy in [2]. The crucial point consists in describing collision effects on the distribution $f^{i}$ by means of a unique collision operator of relaxation type, able to collect all interactions involving species $i$. Specifically, we propose our BGK model in the form

$$
\frac{\partial f^{i}}{\partial t}+\mathbf{v} \cdot \nabla_{\mathbf{x}} f^{i}=\nu^{i}\left(\mathcal{M}^{i}-f^{i}\right), \quad i=1, \ldots, N,
$$

where $\nu^{i}$ are $\mathbf{v}$-independent collision frequencies, and $\mathcal{M}^{i}$ are suitable Maxwellian "attractors" accommodated at "auxiliary" species number densities $\widetilde{n}^{i}$, and at common mass velocity $\widetilde{\mathbf{u}}$ and temperature $\widetilde{T}$

$$
\mathcal{M}^{i}(\mathbf{v}, I)=\frac{\widetilde{n}^{i}}{q^{i}(\widetilde{T})}\left(\frac{m^{i}}{2 \pi \widetilde{T}}\right)^{3 / 2} \mathrm{e}^{-\frac{1}{\widetilde{T}}\left[\frac{m^{i}}{2}|\mathbf{v}-\widetilde{\mathbf{u}}|^{2}+I\right]}, \quad i=1, \ldots, N
$$

This set of $N$ attractors has $N+4$ independent auxiliary parameters, which are $\widetilde{n}^{i}(i=1, \ldots, N)$,

$\widetilde{\mathbf{u}}, \widetilde{T}$, which have to be suitably tuned in order to preserve the basic features of the original Boltzmann 
equations. In particular, here we want to determine the disposable parameters by imposing that our BGK model reproduces the correct $N+4$ collision invariants of the Boltzmann model [12]. Proceeding in this way, our BGK model will automatically share with the Boltzmann kinetic model the same conservation laws. The explicit finding of the auxiliary parameters is explained here below.

- Auxiliary number densities $\widetilde{n}^{i}$

Conservation of species number densities yields the equations

$$
\int_{\mathbb{R}^{3}} \int_{0}^{+\infty} \nu^{i}\left(\mathcal{M}^{i}-f^{i}\right) \varphi^{i}(I) d I d \mathbf{v}=0, \quad i=1, \ldots, N,
$$

that may be cast as

$$
\int_{\mathbb{R}^{3}} \int_{0}^{+\infty} \mathcal{M}^{i}(\mathbf{v}, I) \varphi^{i}(I) d I d \mathbf{v}=n^{i} .
$$

Bearing in mind the definition of $q^{i}$, given in expression (8), and the fact that

$$
\left(\frac{m^{i}}{2 \pi \widetilde{T}}\right)^{3 / 2} \int_{\mathbb{R}^{3}} \mathrm{e}^{-\frac{m^{i}}{2 \widetilde{T}}|\mathbf{v}-\widetilde{\mathbf{u}}|^{2}} d \mathbf{v}=1,
$$

we get

$$
\widetilde{n}^{i}=n^{i} .
$$

Therefore, the auxiliary number densities of the attractors coincide with the actual number densities of the model.

\section{- Auxiliary velocity $\widetilde{u}$}

Conservation of global momentum reads as

$$
\sum_{i=1}^{N} \int_{\mathbb{R}^{3}} \int_{0}^{+\infty} \nu^{i}\left(\mathcal{M}^{i}-f^{i}\right) m^{i} \mathbf{v} \varphi^{i}(I) d I d \mathbf{v}=\mathbf{0} .
$$

Using condition (15) for $\widetilde{n}^{i}$, we obtain

$$
\sum_{i=1}^{N} \nu^{i} m^{i} n^{i} \widetilde{\mathbf{u}}=\sum_{i=1}^{N} \nu^{i} m^{i} n^{i} \mathbf{u}^{i}
$$

so that the auxiliary mass velocity $\widetilde{\mathbf{u}}$ is determined by

$$
\widetilde{\mathbf{u}}=\sum_{i=1}^{N} \nu^{i} m^{i} n^{i} \mathbf{u}^{i} /\left(\sum_{i=1}^{N} \nu^{i} m^{i} n^{i}\right)
$$

Equation (17) is identical to the corresponding one obtained in [2] for gases with discrete energy levels.

- Auxiliary temperature $\widetilde{T}$

Conservation of total energy provides the constraint

$$
\sum_{i=1}^{N} \int_{\mathbb{R}^{3}} \int_{0}^{+\infty} \nu^{i}\left(\mathcal{M}^{i}-f^{i}\right)\left(\frac{1}{2} m^{i}|\mathbf{v}|^{2}+I\right) \varphi^{i}(I) d I d \mathbf{v}=0 .
$$

Recalling the definition of $\chi^{i}$ given in (6), the integral of the distribution function $f^{i}$ appearing in (18) leads to

$$
\sum_{i=1}^{N} \int_{\mathbb{R}^{3}} \int_{0}^{+\infty} \nu^{i} f^{i}(\mathbf{v}, I)\left(\frac{1}{2} m^{i}|\mathbf{v}|^{2}+I\right) \varphi^{i}(I) d I d \mathbf{v}=\sum_{i=1}^{N} \nu^{i}\left(\frac{1}{2} m^{i} n^{i}\left|\mathbf{u}^{i}\right|^{2}+\frac{3}{2} n^{i} T^{i}+\chi^{i}\right) .
$$


Analogously, as concerns the integral of the Maxwellian attractor, we get

$$
\begin{aligned}
\sum_{i=1}^{N} \int_{\mathbb{R}^{3}} \int_{0}^{+\infty} \nu^{i} \mathcal{M}^{i}(\mathbf{v}, I)\left(\frac{1}{2} m^{i}|\mathbf{v}|^{2}+I\right) \varphi^{i}(I) d I d \mathbf{v} \\
=\sum_{i=1}^{N} \nu^{i}\left(\frac{1}{2} m^{i} n^{i}|\widetilde{\mathbf{u}}|^{2}+\frac{3}{2} n^{i} \widetilde{T}+n^{i} \xi^{i}(\widetilde{T})\right)
\end{aligned}
$$

where

$$
\xi^{i}(T)=\frac{\eta^{i}(T)}{q^{i}(T)} .
$$

Therefore, Eq. (18) is a transcendental equation for $\widetilde{T}$, that may be cast as

$$
\frac{3}{2}\left(\sum_{i=1}^{N} \nu^{i} n^{i}\right) \widetilde{T}+\sum_{i=1}^{N} \nu^{i} n^{i} \xi^{i}(\widetilde{T})=\sum_{i=1}^{N} \nu^{i}\left[\frac{1}{2} m^{i} n^{i}\left(\left|\mathbf{u}^{i}\right|^{2}-|\widetilde{\mathbf{u}}|^{2}\right)+\frac{3}{2} n^{i} T^{i}+\chi^{i}\right] .
$$

To proceed, we have to prove that equation (20) admits a unique solution for $\widetilde{T}$. This will be the objective of the following lemma.

Lemma 3.1 The auxiliary temperature $\widetilde{T}$ is uniquely determined by equation (20).

Proof. We start by writing Eq. (20) in the form $F(\widetilde{T})=\Lambda$, where

$$
\begin{aligned}
& F(\widetilde{T})=\sum_{i=1}^{N} \nu^{i} n^{i}\left(\frac{3}{2} \widetilde{T}+\xi^{i}(\widetilde{T})\right), \\
& \Lambda=\sum_{i=1}^{N} \nu^{i}\left[\frac{1}{2} m^{i} n^{i}\left(\left|\mathbf{u}^{i}\right|^{2}-|\widetilde{\mathbf{u}}|^{2}\right)+\frac{3}{2} n^{i} T^{i}+\chi^{i}\right],
\end{aligned}
$$

and observe that $\Lambda$ is independent of the unknown $\widetilde{T}$, since it is known in terms of the actual fields $n^{i}, \mathbf{u}^{i}$ and $T^{i}$. Moreover, $\Lambda>0$ since, bearing in mind the expression of $\widetilde{\mathbf{u}}$ given by Eq. (17), it can be proved that (see Lemma 3.1 in [2] for a similar proof)

$$
\sum_{i=1}^{N} \nu^{i} m^{i} n^{i}\left(\left|\mathbf{u}^{i}\right|^{2}-|\widetilde{\mathbf{u}}|^{2}\right)=\frac{1}{2} \frac{1}{\sum_{j=1}^{N} \nu^{j} m^{j} n^{j}} \sum_{i=1}^{N} \sum_{j=1}^{N} \nu^{i} \nu^{j} m^{i} m^{j} n^{i} n^{j}\left|\mathbf{u}^{i}-\mathbf{u}^{j}\right|^{2} \geq 0 .
$$

Next, we will prove that $F$ is a strictly increasing function of $\widetilde{T} \in[0,+\infty[$. Indeed, we have that

$$
F^{\prime}(\widetilde{T})=\frac{3}{2} \sum_{i=1}^{N} \nu^{i} n^{i}+\sum_{i=1}^{N} \nu^{i} n^{i} \sigma^{i}(\widetilde{T})
$$

where

$$
\sigma^{i}(\widetilde{T})=\left(\xi^{i}\right)^{\prime}(\widetilde{T})=\frac{\left(\eta^{i}\right)^{\prime} q^{i}-\eta^{i}\left(q^{i}\right)^{\prime}}{\left(q^{i}\right)^{2}}(\widetilde{T}) .
$$

Since $\left(q^{i}\right)^{\prime}$ and $\left(\eta^{i}\right)^{\prime}$ are given by

$$
\left(q^{i}\right)^{\prime}(\widetilde{T})=\frac{1}{(\widetilde{T})^{2}} \int_{0}^{+\infty} I \varphi^{i}(I) \mathrm{e}^{-I / \widetilde{T}} d I, \quad\left(\eta^{i}\right)^{\prime}(\widetilde{T})=\frac{1}{(\widetilde{T})^{2}} \int_{0}^{+\infty} I^{2} \varphi^{i}(I) \mathrm{e}^{-I / \widetilde{T}} d I,
$$


we can write

$$
\begin{aligned}
\sigma^{i}(\widetilde{T}) & =\frac{1}{(\widetilde{T})^{2}\left(q^{i}\right)^{2}(\widetilde{T})} \int_{0}^{+\infty} \int_{0}^{+\infty} \varphi^{i}(I) \varphi^{i}(K) \mathrm{e}^{-I / \widetilde{T}} \mathrm{e}^{-K / \widetilde{T}}\left(I^{2}-I K\right) d I d K \\
& =\frac{1}{2(\widetilde{T})^{2}\left(q^{i}\right)^{2}(\widetilde{T})} \int_{0}^{+\infty} \int_{0}^{+\infty} \varphi^{i}(I) \varphi^{i}(K) \mathrm{e}^{-I / \widetilde{T}} \mathrm{e}^{-K / \widetilde{T}}(I-K)^{2} d I d K
\end{aligned}
$$

where last equality is obtained by exchanging variables $I$ and $K$, since $I$ and $K$ have the same range of variability, so that

$$
\sigma^{i}(\widetilde{T}) \geq 0
$$

Therefore, $F^{\prime}(\widetilde{T})>0$, for $\left.\widetilde{T} \in\right] 0,+\infty[$, and $F$ is strictly increasing for $\widetilde{T} \geq 0$.

Finally, we will prove that $F(\widetilde{T})$ ranges from 0 to $+\infty$. From expression $(21)$, one can easily check that

$$
\lim _{\widetilde{T} \rightarrow+\infty} F(\widetilde{T})=+\infty .
$$

Additionally, we have that

$$
\lim _{\widetilde{T} \rightarrow 0^{+}} F(\widetilde{T})=\lim _{\widetilde{T} \rightarrow 0^{+}} \sum_{i=1}^{N} \nu^{i} n^{i} \xi^{i}(\widetilde{T})=\lim _{\widetilde{T} \rightarrow 0^{+}} \sum_{i=1}^{N} \nu^{i} n^{i} \frac{\int_{0}^{+\infty} I \mathrm{e}^{-I / \widetilde{T}} \varphi^{i}(I) d I}{\int_{0}^{+\infty} \mathrm{e}^{-I / \widetilde{T}} \varphi^{i}(I) d I},
$$

and changing the integration variable, setting $K=I / \widetilde{T}$, we get

$$
\lim _{\widetilde{T} \rightarrow 0^{+}} F(\widetilde{T})=\lim _{\widetilde{T} \rightarrow 0^{+}} \sum_{i=1}^{N} \nu^{i} n^{i} \widetilde{T} \frac{\int_{0}^{+\infty} K \mathrm{e}^{-K} \varphi^{i}(K \widetilde{T}) d K}{\int_{0}^{+\infty} \mathrm{e}^{-K} \varphi^{i}(K \widetilde{T}) d K}
$$

and thus

$$
\lim _{\widetilde{T} \rightarrow 0^{+}} F(\widetilde{T})=0 .
$$

In conclusion, function $F(\widetilde{T})$ is strictly increasing and ranges from 0 to $+\infty$, therefore equation (20) admits a unique solution. The proof is then complete.

As a consequence of Eqs. (15), (17) and Lemma 3.1, all auxiliary parameters of our BGK model are well defined and the construction of the model is complete. In particular, the constraint coming from the energy conservation does not give an explicit expression for $\widetilde{T}$, and one has to solve numerically the transcendental equation $F(\widetilde{T})=\Lambda$, with $F(\widetilde{T})$ and $\Lambda$ given in (21) and (22), respectively. See paper [2] where, for a similar problem, the authors have checked that a bisection method is enough to find the solution, since $F$ is monotone.

A particular case of interest for which it is possible to determine $\widetilde{T}$ explicitly is considered in the following lemma.

Lemma 3.2 When the mixture is composed by polytropic gases, then equation (20) gives

$$
\widetilde{T}=\frac{\Lambda}{\sum_{i=1}^{N} \nu^{i} n^{i}\left(\alpha_{i}+\frac{5}{2}\right)}
$$


Thus, if all gases are diatomic, one has

$$
\widetilde{T}=\frac{2}{5} \frac{\Lambda}{\sum_{i=1}^{N} \nu^{i} n^{i}} .
$$

Proof. If the mixture constituents are polytropic gases, then the energy measure is given by (2) and we obtain, see (11) and (19),

$$
\eta^{i}(\widetilde{T})=\left(\alpha_{i}+1\right) \widetilde{T} q^{i}(\widetilde{T}), \quad \xi^{i}(\widetilde{T})=\left(\alpha_{i}+1\right) \widetilde{T} .
$$

Therefore equation (20) becomes linear in the unknown $\widetilde{T}$ providing explicitly $\widetilde{T}$ as stated in (31). Additionally, for diatomic molecules we have $\alpha_{i}=0$, for $i=1, \ldots, n$, and $\widetilde{T}$ is given by (32).

We conclude this section by referring that, in spatial homogeneous conditions, an $\mathcal{H}$-theorem can be established for the BGK system (12) in terms of the same $\mathcal{H}$-function of the exact Boltzmann equation for the mixture considered in this section, namely for

$$
\mathcal{H}(t)=\sum_{i=1}^{N} \int_{\mathbb{R}^{3}} \int_{0}^{+\infty} f^{i}(\mathbf{v}, I) \log \left(f^{i}\right) \varphi^{i}(I) d I d \mathbf{v} .
$$

We omit here the details, since a similar result will be provided in the next Section 4 for a chemically reactive mixture.

\section{Reacting mixture}

We consider a mixture of four polyatomic species whose particles are subjected to elastic collisions and reactive collisions obeying the bimolecular and reversible chemical reaction

$$
A^{1}+A^{2} \rightleftharpoons A^{3}+A^{4} .
$$

Each species $A^{i}$, with $i=1, \ldots, 4$, has particle mass $m^{i}$ and energy of chemical link $E^{i}$. Collision equilibria of the Boltzmann equations are again Maxwellian distributions with a common velocity and a common temperature, which are those of the mixture, as in (7), but, in addition, the species number densities and mixture temperature fulfill the following mass action law

$$
\frac{n^{1} n^{2}}{n^{3} n^{4}}=\left(\frac{m^{1} m^{2}}{m^{3} m^{4}}\right)^{3 / 2} \mathrm{e}^{\Delta E / T} \frac{q^{1}(T) q^{2}(T)}{q^{3}(T) q^{4}(T)}
$$

where $\Delta E=E^{3}+E^{4}-E^{1}-E^{2}$ is the reaction heat of the chemical reaction. We assume that $\Delta E>0$ so that the forward reaction, $A^{1}+A^{2} \rightarrow A^{3}+A^{4}$, is endothermic. Moreover, we note that single number densities are not preserved by the chemical reaction, and we have preservation only of suitable combinations of them, such as $n^{1}+n^{3}, n^{1}+n^{4}, n^{2}+n^{4}$. Collision invariants for the Boltzmann model are thus 7 , corresponding to three independent sums of number densities, global momentum, and total energy (kinetic plus internal plus chemical).

\subsection{BGK model for the reacting mixture}

Analogously to the inert case treated in Section 3, we propose a BGK model for the chemically reactive mixture of the kind

$$
\frac{\partial f^{i}}{\partial t}+\mathbf{v} \cdot \nabla_{\mathbf{x}} f^{i}=\nu^{i}\left(\mathcal{M}^{i}-f^{i}\right), \quad i=1, \ldots, 4,
$$


with Maxwellian attractors $\mathcal{M}^{i}$ given by

$$
\mathcal{M}^{i}(\mathbf{v}, I)=\frac{\widetilde{n}^{i}}{q^{i}(\widetilde{T})}\left(\frac{m^{i}}{2 \pi \widetilde{T}}\right)^{3 / 2} \mathrm{e}^{-\frac{1}{T}\left[\frac{m^{i}}{2}|\mathbf{v}-\widetilde{\mathbf{u}}|^{2}+I\right]}, \quad i=1, \ldots, 4 .
$$

This model has 8 free parameters, namely the 4 auxiliary densities $\widetilde{n}^{i}$, the components of the auxiliary velocity $\widetilde{\mathbf{u}}$ and the auxiliary temperature $\widetilde{T}$. Such parameters will be determined by requiring that they fulfill the mass action law, so that

$$
\frac{\widetilde{n}^{1} \widetilde{n}^{2}}{\widetilde{n}^{3} \widetilde{n}^{4}}=\left(\frac{m^{1} m^{2}}{m^{3} m^{4}}\right)^{3 / 2} \mathrm{e}^{\Delta E / \widetilde{T}} \frac{q^{1}(\widetilde{T}) q^{2}(\widetilde{T})}{q^{3}(\widetilde{T}) q^{4}(\widetilde{T})},
$$

and, additionally, by imposing that the BGK model has the same collision invariants of the Boltzmann model, corresponding to the preservation of three independent combinations of number densities, of global momentum and of total energy. In this way, we have 8 unknowns and 8 constraints resulting from the seven conservation laws and from the mass action law. Accordingly, the unknown parameters will be determined as follows.

\section{- Auxiliary number densities $\widetilde{n}^{i}$}

Conservation of combinations of number densities $n^{1}+n^{3}, n^{1}+n^{4}$ and $n^{2}+n^{4}$ give

$$
\begin{aligned}
& \int_{\mathbb{R}^{3}} \int_{0}^{+\infty} \nu^{i}\left(\mathcal{M}^{i}-f^{i}\right) \varphi^{i}(I) d I d \mathbf{v}+\int_{\mathbb{R}^{3}} \int_{0}^{+\infty} \nu^{j}\left(\mathcal{M}^{j}-f^{j}\right) \varphi^{j}(I) d I d \mathbf{v}=0, \\
& \text { for } \quad(i, j)=(1,3),(1,4),(2,4) .
\end{aligned}
$$

Equations (38) provide

$$
\begin{aligned}
& \nu^{1}\left(\widetilde{n}^{1}-n^{1}\right)+\nu^{3}\left(\widetilde{n}^{3}-n^{3}\right)=0 \\
& \nu^{1}\left(\widetilde{n}^{1}-n^{1}\right)+\nu^{4}\left(\widetilde{n}^{4}-n^{4}\right)=0 \\
& \nu^{2}\left(\widetilde{n}^{2}-n^{2}\right)+\nu^{4}\left(\widetilde{n}^{4}-n^{4}\right)=0
\end{aligned}
$$

and consequently we immediately get the constraints

$$
\sum_{i=1}^{4} \nu^{i}\left(\widetilde{n}^{i}-n^{i}\right)=0, \quad \sum_{i=1}^{4} \nu^{i} m^{i}\left(\widetilde{n}^{i}-n^{i}\right)=0 .
$$

Moreover, a suitable combination of (39)-(41) provides the further constraint

$$
\sum_{i=1}^{4} \nu^{i} E^{i}\left(\widetilde{n}^{i}-n^{i}\right)=-\Delta E \nu^{1}\left(\widetilde{n}^{1}-n^{1}\right)
$$

Equations (39)-(41) allow to express three auxiliary number densities in terms of the fourth one, as follows

$$
\begin{aligned}
& \widetilde{n}^{2}=n^{2}+\frac{\nu^{1}}{\nu^{2}}\left(\widetilde{n}^{1}-n^{1}\right) \\
& \widetilde{n}^{3}=n^{3}-\frac{\nu^{1}}{\nu^{3}}\left(\widetilde{n}^{1}-n^{1}\right) \\
& \widetilde{n}^{4}=n^{4}-\frac{\nu^{1}}{\nu^{4}}\left(\widetilde{n}^{1}-n^{1}\right) .
\end{aligned}
$$

In compact form we may write

$$
\widetilde{n}^{i}=n^{i}+\frac{\lambda^{i}}{\nu^{i}} \nu^{1}\left(\widetilde{n}^{1}-n^{1}\right), \quad i=1, \ldots, 4,
$$


where $\lambda^{i}$ are the stoichiometric coefficients $\lambda^{1}=\lambda^{2}=1$ and $\lambda^{3}=\lambda^{4}=-1$. Therefore, the auxiliary number densities are explicitly given by expressions (44) in terms of actual macroscopic fields and of $\widetilde{n}^{1}$, that will be determined below, in Lemma 4.1.

\section{- Auxiliary velocity $\widetilde{\mathbf{u}}$}

Conservation of global momentum reads as

$$
\sum_{i=1}^{4} \int_{\mathbb{R}^{3}} \int_{0}^{+\infty} \nu^{i}\left(\mathcal{M}^{i}-f^{i}\right) m^{i} \mathbf{v} \varphi^{i}(I) d \mathbf{v} d I=\mathbf{0}
$$

and provides

$$
\left(\sum_{i=1}^{4} \nu^{i} m^{i} \widetilde{n}^{i}\right) \widetilde{\mathbf{u}}=\sum_{i=1}^{4} \nu^{i} m^{i} n^{i} \mathbf{u}^{i}
$$

This condition, bearing in mind the second constraint of (42), gives the same auxiliary velocity $\widetilde{\mathbf{u}}$ as the one obtained for the inert mixture, that is

$$
\widetilde{\mathbf{u}}=\sum_{i=1}^{4} \nu^{i} m^{i} n^{i} \mathbf{u}^{i} /\left(\sum_{i=1}^{4} \nu^{i} m^{i} n^{i}\right)
$$

\section{- Auxiliary temperature $\widetilde{T}$}

Conservation of total energy (sum of kinetic, internal and chemical) reads as

$$
\sum_{i=1}^{4} \int_{\mathbb{R}^{3}} \int_{0}^{+\infty} \nu^{i}\left(\mathcal{M}^{i}-f^{i}\right)\left(\frac{1}{2} m^{i}|\mathbf{v}|^{2}+I+E^{i}\right) \varphi^{i}(I) d \mathbf{v} d I=0
$$

and provides the condition

$$
\sum_{i=1}^{4} \nu^{i} \widetilde{n}^{i}\left(\frac{1}{2} m^{i}|\widetilde{\mathbf{u}}|^{2}+\frac{3}{2} \widetilde{T}+\xi^{i}(\widetilde{T})+E^{i}\right)-\sum_{i=1}^{4} \nu^{i}\left(\frac{1}{2} m^{i} n^{i}\left|\mathbf{u}^{i}\right|^{2}+\frac{3}{2} n^{i} T^{i}+\chi^{i}+n^{i} E^{i}\right)=0,
$$

where $\chi^{i}$ and $\xi^{i}$ are defined in (6) and (19), respectively. Using constraints (42) and (43), from the above condition we get

$$
\frac{3}{2} \widetilde{T} \sum_{i=1}^{4} \nu^{i} n^{i}+\sum_{i=1}^{4} \nu^{i} \widetilde{n}^{i} \xi^{i}(\widetilde{T})-\Delta E \nu^{1}\left(\widetilde{n}^{1}-n^{1}\right)=Z,
$$

where $Z$ is an explicit combination of the actual macroscopic fields $n^{i}, u^{i}$ and $T^{i}$, independent from the unknown parameters of the BGK model, given by

$$
Z=\frac{1}{4} \frac{1}{\sum_{j=1}^{4} \nu^{j} m^{j} n^{j}} \sum_{i=1}^{4} \sum_{j=1}^{4} \nu^{i} \nu^{j} m^{i} m^{j} n^{i} n^{j}\left|\mathbf{u}^{i}-\mathbf{u}^{j}\right|^{2}+\frac{3}{2} \sum_{i=1}^{4} \nu^{i} n^{i} T^{i}+\sum_{i=1}^{4} \nu^{i} \chi^{i} .
$$

Therefore, Eq. (48) together with the mass action law (37) constitute a system of two coupled transcendental equations for the unknown auxiliary fields $\widetilde{n}^{1}$ and $\widetilde{T}$, and we have to prove that such a system admits a unique solution. This will be the object of the following lemma.

Lemma 4.1 The auxiliary number density $\widetilde{n}^{1}$ and temperature $\widetilde{T}$ are uniquely determined by equations (48) and (37). 
Proof. First, by inserting the expressions of $\widetilde{n}^{2}, \widetilde{n}^{3}, \widetilde{n}^{4}$ provided by Eqs. (44) in condition (48), we are able to determine explicitly $\widetilde{n}^{1}$ as function of $\widetilde{T}$. More precisely, we obtain

$$
\widetilde{n}^{1}(\widetilde{T})=n^{1}+\frac{1}{\nu^{1}} \mathcal{S}(\widetilde{T})
$$

where the source term $\mathcal{S}(\widetilde{T})$ can be expressed in the form

$$
\mathcal{S}(\widetilde{T})=\frac{\mathcal{N}(\widetilde{T})}{\mathcal{D}(\widetilde{T})}
$$

with the numerator $\mathcal{N}(\widetilde{T})$ and the denominator $\mathcal{D}(\widetilde{T})$ being provided by

$$
\begin{aligned}
& \mathcal{N}(\widetilde{T})=Z-\frac{3}{2} \widetilde{T} \sum_{i=1}^{4} \nu^{i} n^{i}-\sum_{i=1}^{4} \nu^{i} n^{i} \xi^{i}(\widetilde{T}), \\
& \mathcal{D}(\widetilde{T})=\sum_{i=1}^{4} \lambda^{i} \xi^{i}(\widetilde{T})-\Delta E .
\end{aligned}
$$

From Eqs. (44), expressions analogous to Eq. (50) also hold for the auxiliary densities of other species $i=2,3,4$, so that we have

$$
\widetilde{n}^{i}(\widetilde{T})=n^{i}+\frac{\lambda^{i}}{\nu^{i}} \mathcal{S}(\widetilde{T}), \quad i=1, \ldots, 4 .
$$

Now, by taking into account expressions (54), the mass action law (37) may be written as a transcendental equation for the auxiliary temperature $\widetilde{T}$ in the form

$$
\mathcal{G}(\widetilde{T})=\left(\frac{m^{3} m^{4}}{m^{1} m^{2}}\right)^{3 / 2}
$$

where the function $\mathcal{G}(\widetilde{T})$ is defined by

$$
\mathcal{G}(\widetilde{T})=\mathrm{e}^{\Delta E / \widetilde{T}} \frac{q^{1}(\widetilde{T}) q^{2}(\widetilde{T})}{q^{3}(\widetilde{T}) q^{4}(\widetilde{T})} \frac{\widetilde{n}^{3}(\widetilde{T}) \widetilde{n}^{4}(\widetilde{T})}{\widetilde{n}^{1}(\widetilde{T}) \widetilde{n}^{2}(\widetilde{T})}
$$

with $\widetilde{n}^{i}(\widetilde{T})$ given by expressions (54). To prove that $\widetilde{T}$ is univocally determined, we will show that Eq. (55) admits a unique positive solution $\widetilde{T}>0$ in the set $\mathscr{B}$ for which all auxiliary number densities $\widetilde{n}^{i}$ are positive, namely in the set

$$
\mathscr{B}=\left\{\widetilde{T}>0: \max \left(-n^{1} \nu^{1},-n^{2} \nu^{2}\right)<\mathcal{S}(\widetilde{T})<\min \left(n^{3} \nu^{3}, n^{4} \nu^{4}\right)\right\} .
$$

We will prove that

(i) the set $\mathscr{B}$ is a connected set (interval) in $\mathbb{R}^{+}$in which the sign of the denominator $\mathcal{D}(\widetilde{T})$ does not change;

(ii) function $\mathcal{G}(\widetilde{T})$ is strictly increasing in $\mathscr{B}$, ranging from 0 to $+\infty$.

Concerning item (i) first, observe that $\mathcal{N}$ is a strictly decreasing function in $\mathbb{R}^{+}$, since

$$
\mathcal{N}^{\prime}(\widetilde{T})=-\frac{3}{2} \sum_{i=1}^{4} \nu^{i} n^{i}-\sum_{i=1}^{4} \nu^{i} n^{i} \sigma^{i}(\widetilde{T}),
$$

with $\sigma^{i}(\widetilde{T}) \geq 0$ for $\widetilde{T} \in \mathbb{R}^{+}$, see (26) and (27). Moreover, it can be checked that

$$
\lim _{\widetilde{T} \rightarrow 0^{+}} \mathcal{N}(\widetilde{T})=Z>0, \quad \lim _{\widetilde{T} \rightarrow+\infty} \mathcal{N}(\widetilde{T})=-\infty,
$$


see definitions (49), (52), together with expressions (29), (30) for the first assertion and (21), (28) for the second one. Therefore function $\mathcal{N}$ has a unique zero $\widetilde{T}^{\sharp}$ in $\mathbb{R}^{+}$. From definition (51) of $\mathcal{S}(\widetilde{T})$, we conclude that $\widetilde{T}^{\sharp}$ is the unique possible zero of $\mathcal{S}$ in $\mathscr{B}$.

We note that if there exists $\widetilde{T}^{*} \neq \widetilde{T}^{\sharp}$ such that $\mathcal{D}\left(\widetilde{T}^{*}\right)=0$ then $\lim _{\widetilde{T} \rightarrow \widetilde{T}^{*}} \mathcal{S}(\widetilde{T})= \pm \infty$ and such $\widetilde{T}^{*}$ is out of our admissible set $\mathscr{B}$. We may exclude values of $\Delta E$ such that $\mathcal{D}\left(\widetilde{T}^{\sharp}\right)=0$ : in such a particular case $\widetilde{T}=\widetilde{T}^{\sharp}$ would be a trivial solution of Eq. (55).

Moreover, we have that

$$
\mathcal{S}^{\prime}(\widetilde{T})=\frac{\mathcal{N}^{\prime}(\widetilde{T})}{\mathcal{D}(\widetilde{T})}-\mathcal{S}(\widetilde{T}) \frac{\mathcal{D}^{\prime}(\widetilde{T})}{\mathcal{D}(\widetilde{T})}
$$

with $\mathcal{N}^{\prime}(\widetilde{T})$ given by $(58)$ and

$$
\mathcal{D}^{\prime}(\widetilde{T})=\sum_{i=1}^{4} \lambda^{i} \sigma^{i}(\widetilde{T})
$$

Consequently, $\mathcal{S}^{\prime}(\widetilde{T})$ can be written as

$$
\mathcal{S}^{\prime}(\widetilde{T})=-\frac{1}{\mathcal{D}(\widetilde{T})}\left\{\frac{3}{2} \sum_{i=1}^{4} \nu^{i} n^{i}+\sum_{i=1}^{4} \sigma^{i}(\widetilde{T})\left[\nu^{i} n^{i}+\lambda^{i} \mathcal{S}(\widetilde{T})\right]\right\} .
$$

Since the content of the curly brackets in $(61)$ is positive in the set $\mathscr{B}$, the function $\mathcal{S}(\widetilde{T})$ is strictly monotone in any interval contained in $\mathscr{B}$ in which the sign of $\mathcal{D}(\widetilde{T})$ does not change. More precisely, the sign of $\mathcal{S}^{\prime}(\widetilde{T})$ is opposite to the one of $\mathcal{D}(\widetilde{T})$. Therefore, in each "connected subset" of $\mathscr{B}$, the function $\mathcal{S}(\widetilde{T})$ ranges in a monotone way from its (negative) infimum to its (positive) supremum, and there exists $\widetilde{T} \in \mathscr{B}$ such that $\mathcal{S}(\widetilde{T})=0$. Such value of $\widetilde{T}$ is precisely the zero of $\mathcal{N}$. Consequently, $\mathscr{B}$ has a unique "connected subset", which means that $\mathscr{B}$ is an interval in $\mathbb{R}^{+}$in which the sign of the denominator $\mathcal{D}(\widetilde{T})$ does not change.

Concerning item (ii), we come back now to function $\mathcal{G}(\widetilde{T})$ defined in (56) and compute its derivative. We obtain

$$
\mathcal{G}^{\prime}(\widetilde{T})=\mathcal{G}(\widetilde{T})\left\{-\frac{\Delta E}{(\widetilde{T})^{2}}+\sum_{i=1}^{4} \lambda^{i}\left[\frac{\left(q^{i}\right)^{\prime}(\widetilde{T})}{q^{i}(\widetilde{T})}-\frac{\left(\widetilde{n}^{i}\right)^{\prime}(\widetilde{T})}{\widetilde{n}^{i}(\widetilde{T})}\right]\right\} .
$$

Since $\left(q^{i}\right)^{\prime}(\widetilde{T})=\eta^{i}(\widetilde{T}) /(\widetilde{T})^{2}$ and $\left(\widetilde{n}^{i}\right)^{\prime}(\widetilde{T})=\lambda^{i} \mathcal{S}^{\prime}(\widetilde{T}) / \nu^{i}$, we have

$$
\mathcal{G}^{\prime}(\widetilde{T})=\mathcal{G}(\widetilde{T})\left[-\mathcal{S}^{\prime}(\widetilde{T}) \sum_{i=1}^{4} \frac{1}{\nu^{i} \widetilde{n}^{i}(\widetilde{T})}+\frac{\mathcal{D}(\widetilde{T})}{(\widetilde{T})^{2}}\right]
$$

and conclude that $\mathcal{G}^{\prime}(\widetilde{T})$ has constant sign, namely the one of $\mathcal{D}(\widetilde{T})$. Moreover, notice that for $\widetilde{T}$ ranging in the set $\mathscr{B}$, all auxiliary densities $\widetilde{n}^{i}(\widetilde{T})$ are positive, and one among the quantities $\widetilde{n}^{i}(\widetilde{T})$ tends to zero when $\widetilde{T}$ tends to one of the bounds of $\mathscr{B}$, see definitions (54) and (57). For this reason, $\mathcal{G}(\widetilde{T})$ is a monotone function ranging from 0 to $+\infty$ for $\widetilde{T} \in \mathscr{B}$.

In conclusion, equation (55) admits a unique solution and the auxiliary parameter $\widetilde{T}$ is well defined. Therefore $\widetilde{n}^{1}$ and $\widetilde{T}$ are uniquely determined by equations (48) and (37) and the proof of the lemma is completed.

We conclude this subsection by commenting on the proof of Lemma 4.1 in some particular limiting cases of interest for polyatomic mixtures. 
Remark 4.2 The particular situation of gases with equal particle masses would be able to include bimolecular chemical reactions of type $A+A \rightarrow B+B$ only, where $A$ and $B$ are particles of the same physical constituent, but with different levels of excitation (internal energy). In this case, equation (55) becomes $\mathcal{G}(\widetilde{T})=1$, but the proof of existence and uniqueness of its solution does not show any simplification with respect to the one presented in Lemma 4.1.

Remark 4.3 For a mixture of polytropic gases, $\varphi^{i}(I)$ is given by $(2)$ and the function $\mathcal{S}(\widetilde{T})$ defined in (51) becomes

$$
\mathcal{S}(\widetilde{T})=\frac{Z-\widetilde{T} \sum_{i=1}^{4} \nu^{i} n^{i}\left(\frac{5}{2}+\alpha_{i}\right)}{\widetilde{T} \sum_{i=1}^{4} \lambda^{i} \alpha_{i}-\Delta E} .
$$

If, in addition, $\alpha_{i}=\alpha, \forall i$, namely if all molecules have the same number of degrees of freedom, we have

$$
\mathcal{S}(\widetilde{T})=-\frac{1}{\Delta E}\left[Z-\left(\frac{5}{2}+\alpha\right) \widetilde{T} \sum_{i=1}^{4} \nu^{i} n^{i}\right]
$$

and the proof of its monotonicity is trivial, since $\mathcal{S}^{\prime}(\widetilde{T})>0$ in this case. Moreover, the function $\mathcal{G}(\widetilde{T})$ defined in (56) simplifies to

$$
\mathcal{G}(\widetilde{T})=\mathrm{e}^{\Delta E / \widetilde{T}} \frac{\widetilde{n}^{3}(\widetilde{T}) \widetilde{n}^{4}(\widetilde{T})}{\widetilde{n}^{1}(\widetilde{T}) \widetilde{n}^{2}(\widetilde{T})},
$$

and the monotonicity of $\mathcal{G}(\widetilde{T})$ is straightforward, since

$$
\mathcal{G}^{\prime}(\widetilde{T})=\mathcal{G}(\widetilde{T})\left[-\frac{\Delta E}{\widetilde{T}^{2}}-\sum_{i=1}^{4} \lambda^{i} \frac{\left(\widetilde{n}^{i}\right)^{\prime}(\widetilde{T})}{\widetilde{n}^{i}(\widetilde{T})}\right]=-\mathcal{G}(\widetilde{T})\left[\frac{\Delta E}{\widetilde{T}^{2}}+\mathcal{S}^{\prime}(\widetilde{T}) \sum_{i=1}^{4} \frac{1}{\nu^{i} \widetilde{n}^{i}(\widetilde{T})}\right]<0 .
$$

\section{$4.2 \quad \mathcal{H}$-Theorem for the spatially homogeneous evolution}

We consider spatially homogeneous conditions for the reactive mixture of polyatomic gases, and prove an $\mathcal{H}$-theorem for the BGK model described in Subsection 4.1. More specifically, we prove that the $\mathcal{H}$-function of the exact reactive Boltzmann equation of Ref. [12] is also a Ljapounov functional for the BGK model given in Eqs. (35).

Accordingly, we introduce the function

$$
\mathcal{H}(t)=\sum_{i=1}^{4} \int_{\mathbb{R}^{3}} \int_{0}^{+\infty} f^{i}(\mathbf{v}, I) \log \left(\frac{f^{i}}{\left(m^{i}\right)^{3}}\right) \varphi^{i}(I) d I d \mathbf{v},
$$

and prove the following $\mathcal{H}$-theorem.

Proposition 4.4 For all measurable functions $f^{i}(\mathbf{v}, I) \geq 0, i=1,2,3,4$, we have

$$
\mathcal{H}^{\prime}(t) \leq 0, \quad \text { for all } t \geq 0
$$

and

$$
\mathcal{H}^{\prime}(t)=0 \quad \text { iff } \quad f^{i}=\mathcal{M}^{i} \quad \text { for } \quad i=1,2,3,4 .
$$


Proof. The proof follows in general the usual line in kinetic theory [10], but requires a new argument, similarly to what is done in paper [2] for the BGK model studied there. We start by multiplying the BGK equations (35) in the spatial homogeneous case by $\log \left(f^{i} /\left(m^{i}\right)^{3}\right)$, then integrating over $I$ and $\mathbf{v}$ and summing over the index $i$. We obtain

$$
\begin{aligned}
\frac{d \mathcal{H}}{d t}(t)=\sum_{i=1}^{4} & \int_{\mathbb{R}^{3}} \int_{0}^{+\infty} \nu^{i} \mathcal{M}^{i} \log \left(\frac{f^{i}}{\mathcal{M}^{i}}\right)\left(1-\frac{f^{i}}{\mathcal{M}^{i}}\right) \varphi^{i}(I) d I d \mathbf{v} \\
& +\sum_{i=1}^{4} \int_{\mathbb{R}^{3}} \int_{0}^{+\infty} \nu^{i} \log \left(\frac{\mathcal{M}^{i}}{\left(m^{i}\right)^{3}}\right)\left(\mathcal{M}^{i}-f^{i}\right) \varphi^{i}(I) d I d \mathbf{v} .
\end{aligned}
$$

The usual convexity argument applies to the first term on the r.h.s. of Eq. (65), since it is a linear combination with non-negative coefficients of several contributions with the form $(1-x) \log x$. Concerning the second term on the r.h.s. of Eq. (65), we will prove that it vanishes for all $f^{i}, i=1,2,3,4$, and for all $t \geq 0$. In fact, from expressions (37), we have

$$
\log \left(\frac{\mathcal{M}^{i}}{\left(m^{i}\right)^{3}}\right)=\log \left(\widetilde{n}^{i}\right)-\log \left(q^{i}\right)-\frac{3}{2} \log \left(m^{i}\right)-\frac{3}{2} \log (2 \pi \widetilde{T})-\frac{m^{i}}{2 \widetilde{T}}|\mathbf{v}-\widetilde{\mathbf{u}}|^{2}-\frac{I}{\widetilde{T}}
$$

therefore

$$
\begin{aligned}
\sum_{i=1}^{4} \int_{\mathbb{R}^{3}} \int_{0}^{+\infty} \nu^{i} \log \left(\frac{\mathcal{M}^{i}}{\left(m^{i}\right)^{3}}\right)\left(\mathcal{M}^{i}-f^{i}\right) \varphi^{i}(I) d I d \mathbf{v} \\
=\sum_{i=1}^{4} \int_{\mathbb{R}^{3}} \int_{0}^{+\infty} \nu^{i}\left(\mathcal{M}^{i}-f^{i}\right)\left[\log \left(\widetilde{n}^{i}\right)-\log \left(q^{i}\right)-\frac{3}{2} \log \left(m^{i}\right)-\frac{3}{2} \log (2 \pi \widetilde{T})\right] \varphi^{i}(I) d I d \mathbf{v} \\
\quad-\sum_{i=1}^{4} \int_{\mathbb{R}^{3}} \int_{0}^{+\infty} \nu^{i}\left(\mathcal{M}^{i}-f^{i}\right)\left[\frac{m^{i}}{2 \widetilde{T}}|\mathbf{v}-\widetilde{\mathbf{u}}|^{2}+\frac{I}{\widetilde{T}}\right] \varphi^{i}(I) d I d \mathbf{v} .
\end{aligned}
$$

Using the conservation laws (38), (45), (47) and conditions (42) to manipulate the second term on the r.h.s. of the Eq. (67), we obtain

$$
\begin{aligned}
\sum_{i=1}^{4} \int_{\mathbb{R}^{3}} \int_{0}^{+\infty} \nu^{i} \log \left(\frac{\mathcal{M}^{i}}{\left(m^{i}\right)^{3}}\right)\left(\mathcal{M}^{i}-f^{i}\right) \varphi^{i}(I) d I d \mathbf{v} \\
=\sum_{i=1}^{4} \nu^{i}\left(\widetilde{n}^{i}-n^{i}\right)\left[\log \left(\widetilde{n}^{i}\right)-\log \left(q^{i}\right)-\frac{3}{2} \log \left(m^{i}\right)-\frac{3}{2} \log (2 \pi \widetilde{T})\right] \\
\quad+\frac{1}{\widetilde{T}} \sum_{i=1}^{4} \lambda^{i} E^{i} \nu^{i}\left(\widetilde{n}^{1}-n^{1}\right),
\end{aligned}
$$

or, equivalently, bearing in mind (44),

$$
\begin{aligned}
& \sum_{i=1}^{4} \int_{\mathbb{R}^{3}} \int_{0}^{+\infty} \nu^{i} \log \left(\frac{\mathcal{M}^{i}}{\left(m^{i}\right)^{3}}\right)\left(\mathcal{M}^{i}-f^{i}\right) \varphi^{i}(I) d I d \mathbf{v} \\
& \quad=\nu^{1}\left(\widetilde{n}^{1}-n^{1}\right) \log \left[\left(\frac{\widetilde{n}^{1} \widetilde{n}^{2}}{\widetilde{n}^{3} \widetilde{n}^{4}}\right)\left(\frac{q^{3}(\widetilde{T}) q^{4}(\widetilde{T})}{q^{1}(\widetilde{T}) q^{2}(\widetilde{T})}\right)\left(\frac{m^{3} m^{4}}{m^{1} m^{2}}\right)^{3 / 2} \mathrm{e}^{-\Delta E / \widetilde{T}}\right]
\end{aligned}
$$

Using now condition (37), it is straightforward to conclude that

$$
\sum_{i=1}^{4} \int_{\mathbb{R}^{3}} \int_{0}^{+\infty} \nu^{i} \log \left(\frac{\mathcal{M}^{i}}{\left(m^{i}\right)^{3}}\right)\left(\mathcal{M}^{i}-f^{i}\right) \varphi^{i}(I) d I d \mathbf{v}=0
$$

and the proof is complete. 


\section{Transport properties}

We start with our BGK equations (35) for the reactive mixture of polyatomic gases, where the Maxwellian attractors are defined by (36), with the auxiliary fields determined by (44), (46), (48) and (37). We then introduce a convenient scaling for time and space variables in terms of typical reference values. The resulting equations can be written in the form

$$
\frac{\partial f^{i}}{\partial t}+\mathbf{v} \cdot \nabla_{\mathbf{x}} f^{i}=\frac{1}{\varepsilon} \nu^{i}\left(\mathcal{M}^{i}-f^{i}\right), \quad i=1, \ldots, 4,
$$

where the same symbols have been adopted for the dimensionless variables. Here $\varepsilon$ represents a small parameter (the Knudsen number) that governs the transition from the kinetic BGK model to any hydrodynamic regime, in which $\varepsilon \ll 1$.

\subsection{Asymptotic analysis}

We will use the Chapman-Enskog method and search for a solution to the dimensionless BGK equations (71) as a formal expansion in $\varepsilon$ of the form

$$
f^{i}=f^{i(0)}+\varepsilon f^{i(1)}, \quad i=1, \ldots, 4 .
$$

Such expansions for $f^{i}, i=1, \ldots, 4$, induce similar expansions for $n^{i}, \widetilde{\mathbf{u}}^{i}$ and $T^{i}, i=1, \ldots, 4$, and also for all the macroscopic variables, except for the hydrodynamic fields preserved in the reactive mixture that must remain unexpanded. The kinetic temperature $T$ is not conserved by chemical reaction, and thus a similar expansion holds also for $T$. Additionally, the Maxwellian attractors are also expanded,

$$
\mathcal{M}^{i}=\mathcal{M}^{i(0)}+\varepsilon \mathcal{M}^{i(1)}, \quad i=1, \ldots, 4,
$$

and the collisional frequencies $\nu^{i}$ are expanded as well, since they may depend on macroscopic fields, in particular on the number densities $n^{i}$ (see $[2,4]$ ), namely

$$
\nu^{i}=\nu^{i(0)}+\varepsilon \nu^{i(1)}, \quad i=1, \ldots, 4 .
$$

Inserting expansions (72), (73) and (74) in Eqs. (71), we obtain

$$
\begin{aligned}
\frac{\partial f^{i(0)}}{\partial t}+\mathbf{v} \cdot \nabla_{\mathbf{x}} f^{i(0)}+\varepsilon[ & \left.\frac{\partial f^{i(1)}}{\partial t}+\mathbf{v} \cdot \nabla_{\mathbf{x}} f^{i(1)}\right]=\frac{1}{\varepsilon} \nu^{i(0)}\left[\mathcal{M}^{i(0)}-f^{i(0)}\right] \\
& +\nu^{i(1)}\left[\mathcal{M}^{i(0)}-f^{i(0)}\right]+\nu^{i(0)}\left[\mathcal{M}^{i(1)}-f^{i(1)}\right] \\
& +\varepsilon \nu^{i(1)}\left[\mathcal{M}^{i(1)}-f^{i(1)}\right], \quad i=1, \ldots, 4 .
\end{aligned}
$$

\subsection{Zero-order approximation}

At the zero-order of the expansion, equating all terms in $\varepsilon^{-1}$ appearing in Eq. (75), we obtain

$$
\mathcal{M}^{i(0)}-f^{i(0)}=0, \quad i=1, \ldots, 4,
$$

and Eqs. (76) completely determine $f^{i(0)}$. In fact, it results $f^{i(0)}=\mathcal{M}^{i(0)}$, for $i=1, \ldots, 4$, and thus

$$
f^{i(0)}=\frac{\widetilde{n}^{i(0)}}{q^{i}\left(\widetilde{T}^{(0)}\right)}\left(\frac{m^{i}}{2 \pi \widetilde{T}^{(0)}}\right)^{3 / 2} \mathrm{e}^{-\frac{1}{\widetilde{T}^{(0)}}\left[\frac{m^{i}}{2}\left|\mathbf{v}-\widetilde{\mathbf{u}}^{(0)}\right|^{2}+I\right]}, \quad i=1, \ldots, 4 .
$$

Evaluating the moments of $f^{i(0)}$ given by $(77)$, we easily obtain

$$
n^{i(0)}=\widetilde{n}^{i(0)}, \quad \mathbf{u}^{i(0)}=\widetilde{\mathbf{u}}^{(0)}, \quad T^{i(0)}=\widetilde{T}^{(0)}, \quad i=1, \ldots, 4 .
$$


Since $\widetilde{n}^{i(0)}, i=1, \ldots, 4$, and $\widetilde{T}^{(0)}$ are constrained to the mass action law (37), we conclude that

$$
\frac{n^{1(0)} n^{2(0)}}{n^{3(0)} n^{4(0)}}=\left(\frac{m^{1} m^{2}}{m^{3} m^{4}}\right)^{3 / 2} \mathrm{e}^{\Delta E / T^{(0)}} \frac{q^{1}\left(T^{(0)}\right) q^{2}\left(T^{(0)}\right)}{q^{3}\left(T^{(0)}\right) q^{4}\left(T^{(0)}\right)} .
$$

Consequently, the zero-order approximation $f^{i(0)}$ is completely characterized by

$$
f^{i(0)}=\frac{n^{i(0)}}{q^{i}\left(T^{(0)}\right)}\left(\frac{m^{i}}{2 \pi T^{(0)}}\right)^{3 / 2} \mathrm{e}^{-\frac{1}{T^{(0)}}\left[\frac{m^{i}}{2}\left|\mathbf{v}-\mathbf{u}^{(0)}\right|^{2}+I\right]}, \quad i=1, \ldots, 4,
$$

where $n^{i(0)}, i=1, \ldots, 4$, and $T^{(0)}$ are constrained to condition (79). Therefore, the four distributions $f^{i(0)}$ depend on seven free parameters only and define a macroscopic state of a non-diffusive, non-heat conducting and non-viscous reactive mixture.

According to the Chapman-Enskog asymptotic procedure [11], hydrodynamic quantities corresponding to collision invariants of the kinetic model must remain unexpanded, namely formed by zero order terms only. In the present reactive frame, hydrodynamic variables are three independent combinations of number densities, global momentum, and total energy. Therefore we have at first

$$
n^{i}+n^{j}=n^{i(0)}+n^{j(0)}, \quad(i, j)=(1,3),(1,4),(2,4)
$$

and consequently

$$
n=\sum_{i=1}^{4} n^{i(0)}, \quad \rho=\sum_{i=1}^{4} m^{i} n^{i(0)} .
$$

As concerns global momentum, recalling (80) we have

$$
\rho \mathbf{u}=\sum_{i=1}^{4} m^{i} n^{i(0)} \mathbf{u}^{(0)}=\rho \mathbf{u}^{(0)}
$$

thus $\mathbf{u}^{(0)}=\mathbf{u}$, namely species zero-order velocities coincide with the global macroscopic velocity of the mixture. Finally, for total energy we have

$$
\frac{3}{2} n T+\sum_{i=1}^{4} \chi^{i}+\sum_{i=1}^{4} n^{i} E^{i}=\frac{3}{2} n T^{(0)}+\sum_{i=1}^{4} \chi^{i(0)}+\sum_{i=1}^{4} n^{i(0)} E^{i} .
$$

Requirements (81), (83), (84) imply constraints on the corresponding first order corrections, that must vanish. Specifically, from (81) we have

$$
n^{i(1)}+n^{j(1)}=0, \quad(i, j)=(1,3),(1,4),(2,4),
$$

from which we deduce that all species number density corrections have the same modulus, and only the sign has to be properly set as

$$
n^{i(1)}=\lambda^{i} n^{1(1)}, \quad i=1, \ldots, 4 .
$$

From momentum conservation (83) we have a constraint for first order velocities:

$$
\mathbf{u}^{(1)}=\sum_{i=1}^{4} m^{i} n^{i(0)} \mathbf{u}^{i(1)}=\mathbf{0},
$$

while energy relation (84), taking into account (86), provides

$$
\frac{3}{2} n T^{(1)}+\sum_{i=1}^{4} \chi^{i(1)}-n^{1(1)} \Delta E=0
$$


The macroscopic equations at the zero-order accuracy are evolution equations for the species number densities, and conservation equations for the momentum components and total energy of the mixture. Taking into account the previous analysis, equations for number densities read as

$$
\frac{\partial n^{i(0)}}{\partial t}+\nabla_{\mathbf{x}} \cdot\left(n^{i(0)} \mathbf{u}\right)=\lambda^{i} J_{1}, \quad i=1, \ldots, 4,
$$

where from now on we will denote

$$
J_{1}=\nu^{1(0)}\left(\tilde{n}^{1(1)}-n^{1(1)}\right) .
$$

Momentum conservation may be cast as

$$
\frac{\partial \mathbf{u}}{\partial t}+\mathbf{u} \cdot \nabla_{\mathbf{x}} \mathbf{u}+\frac{1}{\rho} \nabla_{\mathbf{x}}\left(n T^{(0)}\right)=\mathbf{0},
$$

while the energy conservation is more complicated since it involves even internal and chemical energies, so it is omitted here.

\subsection{First-order approximation}

At the first order of the expansion, equating all terms in $\varepsilon^{0}$ appearing in Eq. (75) and taking into account conditions (76), we obtain

$$
\frac{\partial f^{i(0)}}{\partial t}+\mathbf{v} \cdot \nabla_{\mathbf{x}} f^{i(0)}=\nu^{i(0)}\left[\mathcal{M}^{i(1)}-f^{i(1)}\right], \quad i=1, \ldots, 4 .
$$

Now, we have to solve the above Eqs. (92) to determine $f^{i(1)}$. We first evaluate the time and space derivatives of $f^{i(0)}$ and then we compute $\mathcal{M}^{i(1)}$.

Concerning the time and space derivatives of $f^{i(0)}$, standard computations lead to

$$
\begin{aligned}
\frac{\partial f^{i(0)}}{\partial t}= & f^{i(0)}\left\{\frac{1}{n^{i(0)}} \frac{\partial n^{i(0)}}{\partial t}-\frac{\xi^{i}\left(T^{(0)}\right)}{\left(T^{(0)}\right)^{2}} \frac{\partial T^{(0)}}{\partial t}-\frac{3}{2 T^{(0)}} \frac{\partial T^{(0)}}{\partial t}\right. \\
& \left.+\frac{1}{\left(T^{(0)}\right)^{2}}\left[\frac{m^{i}}{2}|\mathbf{v}-\mathbf{u}|^{2}+I\right] \frac{\partial T^{(0)}}{\partial t}+\frac{m^{i}}{T^{(0)}}(\mathbf{v}-\mathbf{u}) \cdot \frac{\partial \mathbf{u}}{\partial t}\right\}, \quad i=1, \ldots, 4,
\end{aligned}
$$

and to $(j=1,2,3)$

$$
\begin{aligned}
\frac{\partial f^{i(0)}}{\partial x_{j}}= & f^{i(0)}\left\{\frac{1}{n^{i(0)}} \frac{\partial n^{i(0)}}{\partial x_{j}}-\frac{\xi^{i}\left(T^{(0)}\right)}{\left(T^{(0)}\right)^{2}} \frac{\partial T^{(0)}}{\partial x_{j}}-\frac{3}{2 T^{(0)}} \frac{\partial T^{(0)}}{\partial x_{j}}\right. \\
& \left.+\frac{1}{\left(T^{(0)}\right)^{2}}\left[\frac{m^{i}}{2}|\mathbf{v}-\mathbf{u}|^{2}+I\right] \frac{\partial T^{(0)}}{\partial x_{j}}+\frac{m^{i}}{T^{(0)}}(\mathbf{v}-\mathbf{u}) \cdot \frac{\partial \mathbf{u}}{\partial x_{j}}\right\}, \quad i=1, \ldots, 4,
\end{aligned}
$$

so that

$$
\mathbf{v} \cdot \nabla_{\mathbf{x}} f^{i(0)}=\sum_{j=1}^{3} v_{j} \frac{\partial f^{i(0)}}{\partial x_{j}}
$$

Concerning the computation of $\mathcal{M}^{i(1)}$, the key idea is the following. From the expansion (73) for $\mathcal{M}^{i}$, we can express $\mathcal{M}^{i(1)}$ as the derivative of $\mathcal{M}^{i}$ with respect to $\varepsilon$, evaluated at the zero-order level $(\varepsilon=0)$, that is

$$
\mathcal{M}^{i(1)}=\left[\frac{d \mathcal{M}^{i}}{d \varepsilon}\right]_{\varepsilon=0}, \quad i=1, \ldots, 4
$$


Standard computations lead to

$$
\begin{aligned}
\mathcal{M}^{i(1)}= & f^{i(0)}\left\{\frac{1}{n^{i(0)}} \widetilde{n}^{i(1)}-\frac{\xi^{i}\left(T^{(0)}\right)}{\left(T^{(0)}\right)^{2}} \widetilde{T}^{(1)}-\frac{3}{2 T^{(0)}} \widetilde{T}^{(1)}\right. \\
& \left.+\frac{1}{\left(T^{(0)}\right)^{2}}\left[\frac{m^{i}}{2}|\mathbf{v}-\mathbf{u}|^{2}+I\right] \widetilde{T}^{(1)}+\frac{m^{i}}{T^{(0)}}(\mathbf{v}-\mathbf{u}) \cdot \widetilde{\mathbf{u}}^{(1)}\right\}, \quad i=1, \ldots, 4 .
\end{aligned}
$$

Inserting expressions (93) and (94) of the time and space derivatives of $f^{i(0)}$ in Eq. (92), together with expression (97) of $\mathcal{M}^{i(1)}$, we obtain a formal solution to Eq. (92) in the form

$$
\begin{aligned}
& f^{i(1)}= f^{i(0)}\left\{\frac{1}{n^{i(0)}} \widetilde{n}^{i(1)}+\frac{m^{i}}{T^{(0)}}(\mathbf{v}-\mathbf{u}) \cdot \widetilde{\mathbf{u}}^{(1)}+\frac{1}{T^{(0)}} \widetilde{T}^{(1)}\left[\frac{m^{i}}{2 T^{(0)}}|\mathbf{v}-\mathbf{u}|^{2}+\frac{I}{T^{(0)}}-\frac{3}{2}-\frac{\xi^{i}\left(T^{(0)}\right)}{T^{(0)}}\right]\right\} \\
&-\frac{1}{\nu^{i(0)}} f^{i(0)}\left\{\frac{1}{n^{i(0)}} \frac{\partial n^{i(0)}}{\partial t}+\frac{m^{i}}{T^{(0)}}(\mathbf{v}-\mathbf{u}) \cdot \frac{\partial \mathbf{u}}{\partial t}\right. \\
&\left.\quad+\frac{1}{T^{(0)}} \frac{\partial T^{(0)}}{\partial t}\left[\frac{m^{i}}{2 T^{(0)}}|\mathbf{v}-\mathbf{u}|^{2}+\frac{I}{T^{(0)}}-\frac{3}{2}-\frac{\xi^{i}\left(T^{(0)}\right)}{T^{(0)}}\right]\right\} \\
&-\frac{1}{\nu^{i(0)}} f^{i(0)}\left\{\frac{1}{n^{i(0)}} \nabla_{\mathbf{x}} n^{i(0)} \cdot \mathbf{v}+\frac{m^{i}}{T^{(0)}} \nabla_{\mathbf{x}} \mathbf{u}: \mathbf{v} \otimes(\mathbf{v}-\mathbf{u})\right. \\
&\left.\quad+\frac{1}{T^{(0)}} \nabla_{\mathbf{x}} T^{(0)}\left[\frac{m^{i}}{2 T^{(0)}}|\mathbf{v}-\mathbf{u}|^{2}+\frac{I}{T^{(0)}}-\frac{3}{2}-\frac{\xi^{i}\left(T^{(0)}\right)}{T^{(0)}}\right] \cdot \mathbf{v}\right\}, \quad i=1, \ldots, 4 .
\end{aligned}
$$

Here, $\widetilde{n}^{i(1)}$, for $i=1, \ldots, 4$, and $\widetilde{\mathbf{u}}^{(1)}, \widetilde{T}^{(1)}$ are the first-order corrections to the auxiliary fields. They can be obtained from Eqs (44), (46) and (48) when the asymptotic expansions of the fields are considered. We easily get

$$
\widetilde{n}^{i(1)}=n^{i(1)}+\frac{\lambda^{i}}{\nu^{i(0)}} J_{1}, \quad i=2,3,4
$$

where $J_{1}$ is defined in (90),

$$
\widetilde{\mathbf{u}}^{(1)}=\left(\sum_{i=1}^{4} \nu^{i(0)} m^{i} n^{i(0)}\right)^{-1}\left(\sum_{i=1}^{4} \nu^{i(0)} m^{i} n^{i(0)} \mathbf{u}^{i(1)}\right),
$$

while the computation of $\widetilde{T}^{(1)}$ is more cumbersome and involves even the expansion of $\chi^{i}$ (defined in (6)):

$$
\begin{aligned}
\chi^{i}=\chi^{i(0)}+\varepsilon \chi^{i(1)} & =\int_{\mathbb{R}^{3}} \int_{0}^{+\infty} f^{i(0)}(\mathbf{v}, I) I \varphi^{i}(I) d I d \mathbf{v}+\varepsilon \int_{\mathbb{R}^{3}} \int_{0}^{+\infty} f^{i(1)}(\mathbf{v}, I) I \varphi^{i}(I) d I d \mathbf{v} \\
& =n^{i(0)} \xi^{i}\left(T^{(0)}\right)+\varepsilon \int_{\mathbb{R}^{3}} \int_{0}^{+\infty} f^{i(1)}(\mathbf{v}, I) I \varphi^{i}(I) d I d \mathbf{v},
\end{aligned}
$$

and after some computations we obtain

$$
\begin{aligned}
\widetilde{T}^{(1)}= & \left(\sum_{i=1}^{4} \nu^{i(0)} n^{i(0)}+\frac{2}{3} \sum_{i=1}^{4} \nu^{i(0)} n^{i(0)} \sigma^{i}\left(T^{(0)}\right)\right)^{-1}\left(\sum_{i=1}^{4} \nu^{i(0)} n^{i(0)} T^{i(1)}+\frac{2}{3} \Delta E J_{1}\right. \\
& \left.+\frac{2}{3} \sum_{i=1}^{4} \nu^{i(0)} \chi^{i(1)}-\frac{2}{3} \sum_{i=1}^{4} \nu^{i(0)} \xi^{i}\left(T^{(0)}\right) n^{i(1)}-\frac{2}{3} J_{1} \sum_{i=1}^{4} \lambda^{i} \xi^{i}\left(T^{(0)}\right)\right) .
\end{aligned}
$$


Moreover, the time derivatives of the hydrodynamic variables $n^{i(0)}, \mathbf{u}$ and $T^{(0)}$ appearing in Eqs. (98) are still unknown and should be computed at the zero-order level from the Euler equations (89) and (91) and from mass action law (79). We obtain

$$
\begin{aligned}
& \frac{1}{n^{i(0)}}\left[\frac{\partial n^{i(0)}}{\partial t}+\mathbf{v} \cdot \nabla_{\mathbf{x}}\left(n^{i(0)}\right)\right]=\frac{1}{n^{i(0)}}(\mathbf{v}-\mathbf{u}) \cdot \nabla_{\mathbf{x}}\left(n^{i(0)}\right) \\
& -\nabla_{\mathbf{x}} \cdot \mathbf{u}+\frac{\lambda^{i}}{n^{i(0)}} J_{1}, \quad i=1,2,3,4, \\
& \frac{m^{i}}{T^{(0)}}\left[(\mathbf{v}-\mathbf{u}) \cdot \frac{\partial \mathbf{u}}{\partial t}+\nabla_{\mathbf{x}} \mathbf{u}: \mathbf{v} \otimes(\mathbf{v}-\mathbf{u})\right]=\frac{m^{i}}{T^{(0)}}\left[\nabla_{\mathbf{x}} \mathbf{u}:(\mathbf{v}-\mathbf{u}) \otimes(\mathbf{v}-\mathbf{u})\right. \\
& \left.-\frac{1}{\rho}(\mathbf{v}-\mathbf{u}) \cdot \nabla_{\mathbf{x}}\left(n T^{(0)}\right)\right] \\
& \frac{1}{T^{(0)}}\left[\frac{\partial T^{(0)}}{\partial t}+\mathbf{v} \cdot \nabla_{\mathbf{x}}\left(T^{(0)}\right)\right]=-\left(\Delta E-\sum_{i=1}^{4} \lambda^{i} \xi^{i}\left(T^{(0)}\right)\right)^{-1} T^{(0)} \sum_{i=1}^{4} \frac{1}{n^{i(0)}} J_{1} \\
& +\frac{1}{T^{(0)}}(\mathbf{v}-\mathbf{u}) \cdot \nabla_{\mathbf{x}}\left(T^{(0)}\right) .
\end{aligned}
$$

Alternatively, the conservation law of total energy could be used instead of the mass action law (79), but in that case the resulting expression for the time derivative of $T^{(0)}$ would be more complicated than expression (105). Obviously, both expressions coincide when the explicit representation of $J_{1}$, found here below in (113), is taken into account. Finally, inserting expressions (103)-(105) in Eq. (98), the solution to Eq. (92), at the first-order level, is explicitly given by

$$
\begin{aligned}
f^{i(1)}=f^{i(0)}\left\{\frac{\widetilde{n}^{i(1)}}{n^{i(0)}}+\frac{m^{i}}{T^{(0)}}(\mathbf{v}-\mathbf{u}) \cdot \widetilde{\mathbf{u}}^{(1)}+\frac{\widetilde{T}^{(1)}}{T^{(0)}}\left[\frac{m^{i}}{2 T^{(0)}}|\mathbf{v}-\mathbf{u}|^{2}+\frac{I}{T^{(0)}}-\frac{3}{2}-\frac{\xi^{i}\left(T^{(0)}\right)}{T^{(0)}}\right]\right\} \\
-\frac{1}{\nu^{i(0)}} f^{i(0)}\left\{\frac{1}{n^{i(0)}}(\mathbf{v}-\mathbf{u}) \cdot \nabla_{\mathbf{x}}\left(n^{i(0)}\right)-\nabla_{\mathbf{x}} \cdot \mathbf{u}+\frac{\lambda^{i}}{n^{i(0)}} J_{1}\right. \\
+\frac{m^{i}}{T^{(0)}}\left[\nabla_{\mathbf{x}} \mathbf{u}:(\mathbf{v}-\mathbf{u}) \otimes(\mathbf{v}-\mathbf{u})-\frac{1}{\rho}(\mathbf{v}-\mathbf{u}) \cdot \nabla_{\mathbf{x}}\left(n T^{(0)}\right)\right] \\
+\left[\frac{m^{i}}{2 T^{(0)}}|\mathbf{v}-\mathbf{u}|^{2}+\frac{I}{T^{(0)}}-\frac{3}{2}-\frac{\xi^{i}\left(T^{(0)}\right)}{T^{(0)}}\right] \times\left[\frac{1}{T^{(0)}}(\mathbf{v}-\mathbf{u}) \cdot \nabla_{\mathbf{x}}\left(T^{(0)}\right)\right. \\
\left.\left.-\left(\Delta E-\sum_{i=1}^{4} \lambda^{i} \xi^{i}\left(T^{(0)}\right)\right)^{-1} T^{(0)} \sum_{i=1}^{4} \frac{1}{n^{i(0)}} J_{1}\right]\right\}, \quad i=1, \ldots, 4,
\end{aligned}
$$

where $\widetilde{n}^{i(1)}, \widetilde{\mathbf{u}}^{(1)}$ and $\widetilde{T}^{(1)}$ are given by expressions (99), (100) and (102).

The first order-approximation $f^{i(1)}, i=1, \ldots, 4$, given explicitly in Eq. (106), incorporates all the nonequilibrium effects induced by the chemical reaction and by the thermodynamic forces appearing at the first level of the approximation. Notice that $f^{i(1)}$ is not completely explicit in terms of zero order hydrodynamic quantities, because of the presence of the first order corrections $n^{i(1)}, \mathbf{u}^{i(1)}, T^{i(1)}$ and $\chi^{i(1)}$ in the expressions of auxiliary fields and in $J_{1}$. Such macroscopic quantities could be directly recomputed as proper moments (with respect to velocity or energy variable) of distribution (106) itself, but an 
analytical computation is quite cumbersome, above all because of the presence of exponential functions in the partition function for internal energy and in the mass action law of chemistry. Some items of this procedure will be described in next subsection, relevant to evolution equations for number densities.

Remark 5.1 In the case of polytropic gases with the same number of degrees of freedom, namely if $\varphi^{i}(I)=I^{\alpha}, \forall i$, from (19) we have $\xi^{i}\left(T^{(0)}\right)=(\alpha+1) T^{(0)}$ and, in the last line of (106), the term $\sum_{i=1}^{4} \lambda^{i} \xi^{i}\left(T^{(0)}\right)$ vanishes. Thus, expression (106) for the first order-approximation $f^{i(1)}$ does not present a significant simplification and the structure of the results presented in this section is approximately the same also in the particular case of polytropic gases, with the exception that some expressions become simpler, as it will be pointed out in the sequel.

\subsection{Balance equations for the number densities}

In physical applications involving gas mixtures with simple chemical reactions, scientists are interested above all in the evolution of number densities of all constituents, in order to investigate both their spatial movement (for instance in presence of polluting powders) and their behaviour in time (some gases could almost disappear, and other ones could widely increase their number of particles). In this subsection we derive hydrodynamic balance equations for number densities from our kinetic BGK model.

Since single gases are not preserved by the chemical reaction, even at Euler level evolution equations of numbers of particles contain a proper collision contribution, as already shown in Eq. (89), rewritten again here for convenience,

$$
\frac{\partial n^{i(0)}}{\partial t}+\nabla_{\mathbf{x}} \cdot\left(n^{i(0)} \mathbf{u}\right)=\lambda^{i} J_{1}, \quad \text { with } \quad J_{1}=\nu^{1(0)}\left(\widetilde{n}^{1(1)}-n^{1(1)}\right), \quad i=1, \ldots, 4 .
$$

In order to get a consistent closure for this set of equations, we have to look for an explicit constitutive law for $J_{1}$, or equivalently for the actual and auxiliary density corrections $n^{1(1)}$ and $\widetilde{n}^{1(1)}$.

\section{- Explicit expression of $J_{1}$}

We start by computing the moment $\chi^{i(1)}$, which does not have its counterpart in classical fluid-dynamic equations, since it is a moment with respect to the internal energy variable, typical of polyatomic gases only. With some computations, bearing in mind the definitions of $\xi^{i}\left(T^{(0)}\right)$ and $\sigma^{i}\left(T^{(0)}\right)$ provided in (19) and $(25)$, respectively, we get

$$
\begin{aligned}
\chi^{i(1)} & =\int_{\mathbb{R}^{3}} \int_{0}^{+\infty} f^{i(1)}(\mathbf{v}, I) I \varphi^{i}(I) d I d \mathbf{v} \\
& =\widetilde{n}^{i(1)} \xi^{i}\left(T^{(0)}\right)+n^{i(0)} \sigma^{i}\left(T^{(0)}\right) \widetilde{T}^{(1)}-\frac{1}{\nu^{i(0)}}\left\{\lambda^{i} \xi^{i}\left(T^{(0)}\right)\right. \\
& \left.-\left(\Delta E-\sum_{j=1}^{4} \lambda^{j} \xi^{j}\left(T^{(0)}\right)\right)^{-1}\left(\sum_{j=1}^{4} \frac{1}{n^{j(0)}}\right)\left[T^{(0)}\right]^{2} n^{i(0)} \sigma^{i}\left(T^{(0)}\right)\right\} J_{1} .
\end{aligned}
$$

By substituting this expression into (102), recalling also the relation between $\widetilde{n}^{i(1)}$ and $J_{1}$ given in (99), we get a simpler expression for the auxiliary temperature $\widetilde{T}^{(1)}$ :

$$
\begin{aligned}
\widetilde{T}^{(1)}= & \left(\sum_{i=1}^{4} \nu^{i(0)} n^{i(0)}\right)^{-1}\left\{\sum_{i=1}^{4} \nu^{i(0)} n^{i(0)} T^{i(1)}+\frac{2}{3}\left[\Delta E-\sum_{i=1}^{4} \lambda^{i} \xi^{i}\left(T^{(0)}\right)\right.\right. \\
& \left.\left.+\left(\Delta E-\sum_{i=1}^{4} \lambda^{i} \xi^{i}\left(T^{(0)}\right)\right)^{-1}\left(\sum_{i=1}^{4} \frac{1}{n^{i(0)}}\right)\left[T^{(0)}\right]^{2}\left(\sum_{i=1}^{4} n^{i(0)} \sigma^{i}\left(T^{(0)}\right)\right)\right] J_{1}\right\},
\end{aligned}
$$


showing that $\widetilde{T}^{(1)}$ depends, besides of course on zero-order hydrodynamic fields, also on $J_{1}$ and on single temperature corrections $T^{i(1)}$.

Concerning the first-order temperatures $T^{i(1)}$, they may be recovered as suitable moments of the distribution functions (106). Indeed, notice that

$$
m^{i} \int_{\mathbb{R}^{3}} \int_{0}^{+\infty}|\mathbf{v}-\mathbf{u}|^{2} f^{i(1)}(\mathbf{v}, I) \varphi^{i}(I) d I d \mathbf{v}=3\left[\frac{d\left(n^{i} T^{i}\right)}{d \varepsilon}\right]_{\varepsilon=0}=3\left(n^{i(1)} T^{(0)}+n^{i(0)} T^{i(1)}\right),
$$

therefore

$$
\begin{aligned}
n^{i(0)} T^{i(1)}= & -n^{i(1)} T^{(0)}+\frac{1}{3} m^{i} \int_{\mathbb{R}^{3}} \int_{0}^{+\infty}|\mathbf{v}-\mathbf{u}|^{2} f^{i(1)}(\mathbf{v}, I) \varphi^{i}(I) d I d \mathbf{v} \\
= & -n^{i(1)} T^{(0)}+\widetilde{n}^{i(1)} T^{(0)}+n^{i(0)} \widetilde{T}^{(1)}-\frac{1}{\nu^{i(0)}}\left\{-n^{i(0)} T^{(0)} \nabla_{\mathbf{x}} \cdot \mathbf{u}+\lambda^{i} T^{(0)} J_{1}\right. \\
& \left.+\frac{5}{3} n^{i(0)} T^{(0)} \nabla_{\mathbf{x}} \cdot \mathbf{u}-\left(\Delta E-\sum_{i=1}^{4} \lambda^{i} \xi^{i}\left(T^{(0)}\right)\right)^{-1}\left(\sum_{j=1}^{4} \frac{1}{n^{j(0)}}\right) n^{i(0)}\left[T^{(0)}\right]^{2} J_{1}\right\},
\end{aligned}
$$

that may be equivalently cast as

$$
n^{i(0)}\left[T^{i(1)}-\widetilde{T}^{(1)}\right]=-\frac{1}{\nu^{i(0)}}\left\{\frac{2}{3} n^{i(0)} T^{(0)} \nabla_{\mathbf{x}} \cdot \mathbf{u}-\left(\Delta E-\sum_{i=1}^{4} \lambda^{i} \xi^{i}\left(T^{(0)}\right)\right)^{-1}\left(\sum_{j=1}^{4} \frac{1}{n^{j(0)}}\right) n^{i(0)}\left[T^{(0)}\right]^{2} J_{1}\right\} .
$$

By inserting into this equation the expression of $\widetilde{T}^{(1)}$ given in (109) we obtain

$$
\begin{aligned}
n^{i(0)} & {\left[T^{i(1)}-\left(\sum_{h=1}^{4} \nu^{h(0)} n^{h(0)}\right)^{-1} \sum_{j=1}^{4} \nu^{j(0)} n^{j(0)} T^{j(1)}\right] } \\
= & \frac{2}{3} n^{i(0)}\left(\sum_{j=1}^{4} \nu^{j(0)} n^{j(0)}\right)^{-1}\left[\Delta E-\sum_{j=1}^{4} \lambda^{j} \xi^{j}\left(T^{(0)}\right)+\left(\Delta E-\sum_{j=1}^{4} \lambda^{j} \xi^{j}\left(T^{(0)}\right)\right)^{-1}\left(\sum_{j=1}^{4} \frac{1}{n^{j(0)}}\right)\left[T^{(0)}\right]^{2}\right. \\
& \left.\times\left(\sum_{j=1}^{4} n^{j(0)} \sigma^{j}\left(T^{(0)}\right)\right)\right] J_{1}-\frac{n^{i(0)}}{\nu^{i(0)}}\left[\frac{2}{3} T^{(0)} \nabla_{\mathbf{x}} \cdot \mathbf{u}-\left(\Delta E-\sum_{j=1}^{4} \lambda^{j} \xi^{j}\left(T^{(0)}\right)\right)^{-1}\left(\sum_{j=1}^{4} \frac{1}{n^{j(0)}}\right)\left[T^{(0)}\right]^{2} J_{1}\right] .
\end{aligned}
$$

We note that a suitable linear combination of the four left hand sides of Eqs. (112), one for each species $i$, vanishes. Precisely, if we multiply by $\nu^{i(0)}$ the $i$-th left hand side and then sum over species $i=1, \ldots, 4$, we get

$$
\begin{aligned}
& \sum_{i=1}^{4} \nu^{i(0)} n^{i(0)}\left[T^{i(1)}-\left(\sum_{h=1}^{4} \nu^{h(0)} n^{h(0)}\right)^{-1} \sum_{j=1}^{4} \nu^{j(0)} n^{j(0)} T^{j(1)}\right] \\
& =\left(\sum_{h=1}^{4} \nu^{h(0)} n^{h(0)}\right)^{-1}\left[\sum_{i=1}^{4} \sum_{h=1}^{4} \nu^{i(0)} \nu^{h(0)} n^{i(0)} n^{h(0)} T^{i(1)}-\sum_{i=1}^{4} \sum_{j=1}^{4} \nu^{i(0)} \nu^{j(0)} n^{i(0)} n^{j(0)} T^{j(1)}\right]=0 .
\end{aligned}
$$

Consequently, the same combination must vanish when we consider the right hand sides of Eqs. (112), 
and therefore

$$
\begin{gathered}
\frac{2}{3}\left[\Delta E-\sum_{j=1}^{4} \lambda^{j} \xi^{j}\left(T^{(0)}\right)+\left(\Delta E-\sum_{j=1}^{4} \lambda^{j} \xi^{j}\left(T^{(0)}\right)\right)^{-1}\left(\sum_{j=1}^{4} \frac{1}{n^{j(0)}}\right)\left[T^{(0)}\right]^{2}\left(\sum_{j=1}^{4} n^{j(0)} \sigma^{j}\left(T^{(0)}\right)\right)\right] J_{1} \\
-n\left[\frac{2}{3} T^{(0)} \nabla_{\mathbf{x}} \cdot \mathbf{u}-\left(\Delta E-\sum_{j=1}^{4} \lambda^{j} \xi^{j}\left(T^{(0)}\right)\right)^{-1}\left(\sum_{j=1}^{4} \frac{1}{n^{j(0)}}\right)\left[T^{(0)}\right]^{2} J_{1}\right]=0,
\end{gathered}
$$

and this provides an explicit expression of $J_{1}$ in terms of zero-order macroscopic fields:

$$
\begin{aligned}
J_{1}= & {\left[\left(\Delta E-\sum_{j=1}^{4} \lambda^{j} \xi^{j}\left(T^{(0)}\right)\right)^{2}+\left(\sum_{j=1}^{4} \frac{1}{n^{j(0)}}\right)\left[T^{(0)}\right]^{2}\left(\frac{3}{2} n+\sum_{j=1}^{4} n^{j(0)} \sigma^{j}\left(T^{(0)}\right)\right)\right]^{-1} } \\
& \times\left(\Delta E-\sum_{j=1}^{4} \lambda^{j} \xi^{j}\left(T^{(0)}\right)\right) n T^{(0)} \nabla_{\mathbf{x}} \cdot \mathbf{u} .
\end{aligned}
$$

This completes the closure of the Euler equations given in (107) for the evolution of the number densities $n^{i(0)}$. Notice that $J_{1}$, and therefore also the number densities corrections, depend on the divergence of the mean velocity $\mathbf{u}$ only, and not on gradients of densities or of temperature.

\section{- Explicit expression of $n^{i(1)}$}

Even with the explicit expression at hand for $J_{1}$, given in (113), it is not easy to make the density corrections $n^{i(1)}$ explicit. To this end, first we use (86), which gives $n^{i(1)}=\lambda^{i} n^{1(1)}$, and thus we look for an explicit expression for $n^{1(1)}$ only. Then we resort to the constraint (88), in which we insert expression (108) for $\chi^{i(1)}$ and we use the fact that $\widetilde{n}^{i(1)}=\lambda^{i}\left(n^{1(1)}+J_{1} / \nu^{i(0)}\right)$, which results from (99). We get

$$
\begin{aligned}
(\Delta E & \left.-\sum_{j=1}^{4} \lambda^{j} \xi^{j}\left(T^{(0)}\right)\right) n^{1(1)}=\frac{3}{2} n T^{(1)}+\sum_{i=1}^{4} n^{i(0)} \sigma^{i}\left(T^{(0)}\right) \widetilde{T}^{(1)} \\
& +\left(\Delta E-\sum_{j=1}^{4} \lambda^{j} \xi^{j}\left(T^{(0)}\right)\right)^{-1}\left(\sum_{j=1}^{4} \frac{1}{n^{j(0)}}\right)\left[T^{(0)}\right]^{2} \sum_{i=1}^{4} \frac{n^{i(0)} \sigma^{i}\left(T^{(0)}\right)}{\nu^{i(0)}} J_{1} .
\end{aligned}
$$

On the other hand, the mass action law for the auxiliary parameters given in (37) may be cast as

$$
\sum_{i=1}^{4} \lambda^{i} \log \left(\widetilde{n}^{i}\right)=\frac{3}{2} \log \left(\frac{m^{1} m^{2}}{m^{3} m^{4}}\right)+\frac{\Delta E}{\widetilde{T}}+\sum_{i=1}^{4} \lambda^{i} \log \left(q^{i}(\widetilde{T})\right)
$$

and its first order terms provide

$$
\left(\sum_{i=1}^{4} \frac{1}{n^{i(0)}}\right) n^{1(1)}=-\frac{1}{\left[T^{(0)}\right]^{2}}\left(\Delta E-\sum_{j=1}^{4} \lambda^{j} \xi^{j}\left(T^{(0)}\right)\right) \widetilde{T}^{(1)}-\left(\sum_{i=1}^{4} \frac{1}{\nu^{i(0)} n^{i(0)}}\right) J_{1} .
$$

Moreover, from temperature definition (5) and from (110), we deduce $n T^{(1)}=\sum_{i=1}^{4} n^{i(0)} T^{i(1)}$. Therefore, from (111), we obtain

$$
n T^{(1)}=n \widetilde{T}^{(1)}-\left(\sum_{i=1}^{4} \frac{n^{i(0)}}{\nu^{i(0)}}\right)\left\{\frac{2}{3} T^{(0)} \nabla_{\mathbf{x}} \cdot \mathbf{u}-\left(\Delta E-\sum_{j=1}^{4} \lambda^{j} \xi^{j}\left(T^{(0)}\right)\right)^{-1}\left(\sum_{j=1}^{4} \frac{1}{n^{j(0)}}\right)\left[T^{(0)}\right]^{2} J_{1}\right\} .
$$


Notice that (114), (115), (116) provide three independent relations for the three unknown fields $n^{1(1)}$, $T^{(1)}, \widetilde{T}^{(1)}$. From (116) we get

$$
\begin{aligned}
\frac{3}{2} n T^{(1)}+ & \sum_{i=1}^{4} n^{i(0)} \sigma^{i}\left(T^{(0)}\right) \widetilde{T}^{(1)}=\left(\frac{3}{2} n+\sum_{i=1}^{4} n^{i(0)} \sigma^{i}\left(T^{(0)}\right)\right) \widetilde{T}^{(1)}-\left(\sum_{i=1}^{4} \frac{n^{i(0)}}{\nu^{i(0)}}\right) T^{(0)} \nabla_{\mathbf{x}} \cdot \mathbf{u} \\
& +\frac{3}{2}\left(\Delta E-\sum_{j=1}^{4} \lambda^{j} \xi^{j}\left(T^{(0)}\right)\right)^{-1}\left(\sum_{i=1}^{4} \frac{n^{i(0)}}{\nu^{i(0)}}\right)\left(\sum_{j=1}^{4} \frac{1}{n^{j(0)}}\right)\left[T^{(0)}\right]^{2} J_{1}
\end{aligned}
$$

and from (115) we obtain an expression for $\widetilde{T}^{(1)}$. Inserting both expressions into (114), one ends up with

$$
\begin{aligned}
n^{1(1)}= & {\left[\left(\Delta E-\sum_{j=1}^{4} \lambda^{j} \xi^{j}\left(T^{(0)}\right)\right)^{2}+\left(\sum_{j=1}^{4} \frac{1}{n^{j(0)}}\right)\left[T^{(0)}\right]^{2}\left(\frac{3}{2} n+\sum_{j=1}^{4} n^{j(0)} \sigma^{j}\left(T^{(0)}\right)\right)\right]^{-1} } \\
& \times\left\{-\left(\Delta E-\sum_{j=1}^{4} \lambda^{j} \xi^{j}\left(T^{(0)}\right)\right)\left(\sum_{i=1}^{4} \frac{n^{i(0)}}{\nu^{i(0)}}\right) T^{(0)} \nabla_{\mathbf{x}} \cdot \mathbf{u}+\left[T^{(0)}\right]^{2}\left[\left(\frac{3}{2} \sum_{i=1}^{4} \frac{n^{i(0)}}{\nu^{i(0)}}\right.\right.\right. \\
& \left.\left.\left.+\sum_{i=1}^{4} \frac{n^{i(0)} \sigma^{i}\left(T^{(0)}\right)}{\nu^{i(0)}}\right)\left(\sum_{i=1}^{4} \frac{1}{n^{i(0)}}\right)-\left(\frac{3}{2} n+\sum_{j=1}^{4} n^{j(0)} \sigma^{j}\left(T^{(0)}\right)\right)\left(\sum_{i=1}^{4} \frac{1}{\nu^{i(0)} n^{i(0)}}\right)\right] J_{1}\right\} .
\end{aligned}
$$

Expression (117), with $J_{1}$ given by (113), gives the explicit representation of $n^{1(1)}$ as well as of all density corrections $n^{i(1)}$, since $n^{i(1)}=\lambda^{i} n^{1(1)}$. It shows that the density corrections turn out to be proportional to the divergence of the mean velocity of the mixture.

In the following remarks, we consider some particular cases in which we obtain simpler expressions.

Remark 5.2 As in Remark 5.1, in the case of polytropic gases with the same number of degrees of freedom, we have $\xi^{i}\left(T^{(0)}\right)=(\alpha+1) T^{(0)}$ and, from $(25)$, we get $\sigma^{i}\left(T^{(0)}\right)=\alpha+1$. Therefore expressions (108) for $\chi^{i(1)}$, (109) for $\widetilde{T}^{(1)}$, and (113) for $J_{1}$ simplify, respectively, to

$$
\begin{gathered}
\chi^{i(1)}=(\alpha+1)\left\{\widetilde{n}^{i(1)} T^{(0)}+n^{i(0)} \widetilde{T}^{(1)}-\frac{T^{(0)}}{\nu^{i(0)}}\left[\lambda^{i}-\frac{1}{\Delta E}\left(\sum_{j=1}^{4} \frac{1}{n^{j(0)}}\right) n^{i(0)} T^{(0)}\right] J_{1}\right\}, \\
\widetilde{T}^{(1)}=\left(\sum_{i=1}^{4} \nu^{i(0)} n^{i(0)}\right)^{-1}\left\{\sum_{i=1}^{4} \nu^{i(0)} n^{i(0)} T^{i(1)}+\frac{2}{3}\left[\Delta E+\frac{1}{\Delta E}\left(\sum_{i=1}^{4} \frac{1}{n^{i(0)}}\right) n\left[T^{(0)}\right]^{2}(\alpha+1)\right] J_{1}\right\}, \\
J_{1}=\left[\Delta E+\frac{1}{\Delta E}\left(\frac{5}{2}+\alpha\right)\left(\sum_{j=1}^{4} \frac{1}{n^{j(0)}}\right) n\left[T^{(0)}\right]^{2}\right]^{-1} n T^{(0)} \nabla_{\mathbf{x}} \cdot \mathbf{u} .
\end{gathered}
$$

Furthermore, the first order number density correction $n^{1(1)}$ given in (117) becomes

$$
\begin{aligned}
n^{1(1)}= & {\left[\Delta E+\frac{1}{\Delta E}\left(\frac{5}{2}+\alpha\right)\left(\sum_{j=1}^{4} \frac{1}{n^{j(0)}}\right) n\left[T^{(0)}\right]^{2}\right]^{-1}\left(\sum_{i=1}^{4} \frac{n^{i(0)}}{\nu^{i(0)}}\right) T^{(0)} } \\
& \times\left\{-\nabla_{\mathbf{x}} \cdot \mathbf{u}+\frac{T^{(0)}}{\Delta E}\left(\frac{5}{2}+\alpha\right)\left[\sum_{i=1}^{4} \frac{1}{n^{i(0)}}-n\left(\sum_{i=1}^{4} \frac{1}{\nu^{i(0)} n^{i(0)}}\right)\left(\sum_{i=1}^{4} \frac{n^{i(0)}}{\nu^{i(0)}}\right)^{-1}\right] J_{1}\right\} .
\end{aligned}
$$


Additionally, in the limiting case of equal collision frequencies, $\nu^{i(0)}=\nu, \forall i$, the previous expression for $n^{1(1)}$ simplifies as

$$
n^{1(1)}=-\left[\Delta E+\frac{1}{\Delta E}\left(\frac{5}{2}+\alpha\right)\left(\sum_{j=1}^{4} \frac{1}{n^{j(0)}}\right) n\left[T^{(0)}\right]^{2}\right]^{-1} \frac{n T^{(0)}}{\nu} \nabla_{\mathbf{x}} \cdot \mathbf{u}=-\frac{J_{1}}{\nu} .
$$

and the auxiliary number density vanishes, that is $\widetilde{n}^{1(1)}=0$, since $\widetilde{n}^{1(1)}=n^{1(1)}+J_{1} / \nu$.

Remark 5.3 Even for mixtures of non-polytropic gases, we may notice that, in the limiting case of equal collision frequencies for all species, namely $\nu^{i(0)}=\nu$, for all $i=1, \ldots, 4$, the coefficient in front of $J_{1}$ appearing in expression (117) would vanish, and the corresponding expression for the density correction becomes $n^{1(1)}=-J_{1} / \nu$. Consequently, also in this case, the auxiliary number density $\widetilde{n}^{1(1)}$ turns out to vanish.

\section{Conclusions and final remarks}

Starting from the Boltzmann model proposed in paper [12] and following the line of paper [2], we have constructed a consistent BGK model for a reactive mixture of polyatomic gases with a continuous structure of internal energies.

The model replaces the Boltzmann collision operator of each species by only one relaxation term which includes reactive and non-reactive contributions. It is consistent with the correct conservation laws and recovers the $\mathcal{H}$-theorem of the Boltzmann model of paper [12]. Additionally, it also reproduces the correct characterization of equilibrium states and mass action law of the chemical reaction.

In the last part of the paper we have developed a Chapman-Enskog asymptotic analysis and computed explicitly the first-order non-equilibrium corrections to the distribution functions. We have also derived the macroscopic evolution equations for the species number densities at the Euler level. Since single components of the mixture are not preserved by the chemical reaction, these evolution equations contain collision contributions associated to the chemical reaction which, in turn, depend on the first-order approximation of the distribution functions. A consistent closure of these equations is obtained by deriving analytical expressions for the reactive production terms.

Although the structure of the relaxation BGK operator of our model is rather simple, the analytical computations are very cumbersome, essentially because of the continuous structure of internal energy and of the presence of exponential functions both in the partition function for the internal energy and in the mass action law for chemical equilibrium. Nevertheless, at the end of the paper, we are able to derive the complete closure of the equations for number densities and also the explicit expression of first-order density corrections. The dependence of densities (and also of temperature, related to densities through the mass action law) on the divergence of macroscopic velocity is in agreement with other asymptotic closures of macroscopic equations obtained in different frames, as a single polyatomic gas with discrete energies or chemically reacting mixtures of monatomic gases, see for instance references $[3,6]$.

On the other hand, in order to present in a simpler way some of the main steps of the construction of the BGK model and of the asymptotic procedure, we discuss, in several remarks along the text, some particular cases in which the computations are easy and the results become clearly expressed.

The results included in this paper are new and provide a rather tractable kinetic model to describe realistic reactive mixtures composed of polyatomic gases. From the physical point of view, our results can be used in applied problems involving multicomponent mixtures undergoing a chemical reaction analogous to the one considered here, i.e. bimolecular and reversible. The interest of a consistent mathematical model to describe polyatomic gases is well known and is documented, for example, in the books $[18,21]$. From the mathematical point of view, the model satisfies several fundamental properties and thus offers good prospectives to investigate mathematical problems of different nature. Starting from 
the results obtained with the asymptotic analysis performed here, the next step could be to proceed to the Navier-Stokes level, deriving macroscopic equations for the hydrodynamic fields of the reactive mixture and computing the transport coefficients of interest. In this reactive frame, the set of Navier-Stokes equations is composed by seven evolution equations, for three suitable combinations of number densities, for global momentum, and for total energy. Equations for number densities will involve even diffusion velocities $\mathbf{u}^{i(1)}$, whose explicit computation is expected to be really complicated, since a suitable Fick matrix and a Soret coefficient should appear. The closure of the momentum equation will require the derivation of a suitable constitutive equation for the first-order pressure tensor, and in particular of its diagonal part $n T^{(1)}$ (usually called dynamical pressure [7, 21]) and of the traceless viscous stress, which is expected to be proportional to the classical strain rate tensor by means of a proper viscosity coefficient. Then, in the energy equation, the evaluation of global heat flux is also required, with computation of the proper conduction coefficient. Notice that in order to write balance equations at Navier-Stokes accuracy for single constituents, and not only for the combinations preserved by chemical reaction, we would need, besides the number density corrections already computed in (117), even a suitable expression for the collision frequencies $\nu^{i(1)}$. These of course would depend on the choice adopted for coefficients $\nu^{i}$ in the BGK model (35). Some examples of frequencies used in numerical simulations of BGK models for mixtures may be found in [4] and in [16]. Of course, the Navier-Stokes closure will require a hard work, because of the above referred computational difficulties, but some preliminary results are already in progress and will be matter of a future work.

\section{Acknowledgments.}

The paper is partially supported by the Italian National Group GNFM of INdAM and by the Portuguese Funds FCT Project UID/MAT/00013/2013. One of the Authors (AJS) thanks the Italian institution for the financial support given in her visiting professor program in Italy.

\section{References}

[1] P. Andries, K. Aoki, B. Perthame, "A consistent BGK-type model for gas mixtures", J. Stat. Phys. 106 (2002), 993-1018.

[2] M. Bisi, M.J. Cáceres, "A BGK relaxation model for polyatomic gas mixtures", Commun. Math. Sci. 14 (2016), 297-325.

[3] M. Bisi, M. Groppi, G. Spiga, "Kinetic Bhatnagar-Gross-Krook model for fast reactive mixtures and its hydrodynamic limit", Phys. Rev. E 81 (2010), 036327 (pp. 1-9).

[4] M. Bisi, S. Lorenzani, "High-frequency sound wave propagation in binary gas mixtures flowing through microchannels", Phys. Fluids 28 (2016), 052003 (pp. 1-21).

[5] M. Bisi, G. Spiga, "On kinetic models for polyatomic gases and their hydrodynamic limits", Ric. Mat., 66 (2017), 113-124.

[6] M. Bisi, G. Spiga, "Hydrodynamic limits of kinetic equations for polyatomic and reactive gases", Commun. Appl. Ind. Math., 8 (2017), 23-42.

[7] M. Bisi, T. Ruggeri, G. Spiga, "Dynamical pressure in a polyatomic gas: interplay between kinetic theory and Extended Thermodynamics", Kinet. Relat. Models, 11 (2018), 71-95.

[8] C. Borgnakke, P.S. Larsen, "Statistical collision model for Monte-Carlo simulation of polyatomic gas mixtures", J. Comput. Phys. 272 (1975), 405-420.

[9] S. Brull, J. Schneider, "Derivation of a BGK model for reacting gas mixtures" Commun. Math. Sci. 12 (2014), 1199-1223. 
[10] C. Cercignani, The Boltzmann Equation and its Applications, Springer, New York, 1988.

[11] S. Chapman, T.G. Cowling, The mathematical theory of non-uniform gases, Cambridge University Press, Cambridge (1970).

[12] L. Desvillettes, R. Monaco, F. Salvarani, "A kinetic model allowing to obtain the energy law of polytropic gases in the presence of chemical reactions", Europ. J. Mech. B/ Fluids 24 (2005), 219236.

[13] V. Giovangigli, Multicomponent flow modeling, Birkhäuser, Boston USA, 1999.

[14] M. Groppi, A. Rossani, G. Spiga, "Kinetic theory of a diatomic gas with reactions of dissociation and recombination through a transition state", J. Phys. A: Math. Gen. 33 (2000), 8819-8833.

[15] M. Groppi, G. Spiga, "Kinetic approach to chemical reactions and inelastic transitions in a rarefied gas", J. Math. Chem., 26 (1999), 197-219.

[16] M. Groppi, G. Spiga, "A Bhatnagar-Gross-Krook-type approach for chemically reacting gas mixtures", Phys. Fluids 16 (2004), 037104, 4273-4284.

[17] G.M. Kremer, M. Pandolfi Bianchi, A.J. Soares, "A relaxation kinetic model for transport phenomena in a reactive flow", Phys. Fluids 18 (2006), 037104, pp. 1-15.

[18] E. Nagnibeda, E. Kustova, Non-equilibrium reacting gas flows, Springer Verlag, Berlin, 2009.

[19] M. Pavić, T. Ruggeri, S. Simić, "Maximum entropy principle for polyatomic gases", Physica A 392 (2013), 1302-1317.

[20] A. Rossani, G. Spiga, "A note on the kinetic theory of chemically reacting gases", Physica A, 272 (1999), 563-573.

[21] T. Ruggeri, M. Sugiyama, Rational extended thermodynamics beyond the monatomic gas, Springer International Publishing, Switzerland, 2015. 